\title{
Synaptic Plasticity Induced by Differential Manipulation of Tonic and Phasic Motoneurons in Drosophila
}

\author{
Nicole A. Aponte-Santiago, Kiel G. Ormerod, Yulia Akbergenova, and ${ }^{\circledR}$ J. Troy Littleton \\ The Picower Institute for Learning and Memory, Department of Biology and Department of Brain and Cognitive Sciences, Massachusetts Institute \\ of Technology, Cambridge, Massachusetts 02139
}

Structural and functional plasticity induced by neuronal competition is a common feature of developing nervous systems. However, the rules governing how postsynaptic cells differentiate between presynaptic inputs are unclear. In this study, we characterized synaptic interactions following manipulations of tonic Ib or phasic Is glutamatergic motoneurons that coinnervate postsynaptic muscles of male or female Drosophila melanogaster larvae. After identifying drivers for each neuronal subtype, we performed ablation or genetic manipulations to alter neuronal activity and examined the effects on synaptic innervation and function at neuromuscular junctions. Ablation of either Ib or Is resulted in decreased muscle response, with some functional compensation occurring in the Ib input when Is was missing. In contrast, the Is terminal failed to show functional or structural changes following loss of the coinnervating Ib input. Decreasing the activity of the Ib or Is neuron with tetanus toxin light chain resulted in structural changes in muscle innervation. Decreased Ib activity resulted in reduced active zone (AZ) number and decreased postsynaptic subsynaptic reticulum volume, with the emergence of filopodial-like protrusions from synaptic boutons of the Ib input. Decreased Is activity did not induce structural changes at its own synapses, but the coinnervating Ib motoneuron increased the number of synaptic boutons and AZs it formed. These findings indicate that tonic Ib and phasic Is motoneurons respond independently to changes in activity, with either functional or structural alterations in the Ib neuron occurring following ablation or reduced activity of the coinnervating Is input, respectively.

Key words: active zone; Drosophila; glutamatergic; motoneuron; neurotransmitter release; synaptic plasticity

Significance Statement

Both invertebrate and vertebrate nervous systems display synaptic plasticity in response to behavioral experiences, indicating that underlying mechanisms emerged early in evolution. How specific neuronal classes innervating the same postsynaptic target display distinct types of plasticity is unclear. Here, we examined whether Drosophila tonic $\mathrm{Ib}$ and phasic Is motoneurons display competitive or cooperative interactions during innervation of the same muscle, or compensatory changes when the output of one motoneuron is altered. We established a system to differentially manipulate the motoneurons and examined the effects of cell type-specific changes to one of the inputs. Our findings indicate $\mathrm{Ib}$ and Is motoneurons respond differently to activity mismatch or loss of the coinnervating input, with the Ib subclass responding robustly compared with Is motoneurons.

Received Apr. 20, 2020; revised June 22, 2020; accepted June 28, 2020.

Author contributions: N.A.A.-S., K.G.O., Y.A., and J.T.L. designed research; N.A.A.-S., K.G.O., Y.A., and J.T.L. performed research; N.A.A.-S., K.G.O., Y.A., and J.T.L. analyzed data; N.A.A.-S. and J.T.L. wrote the paper.

The authors declare no financial or conflicts of interests.

This work was supported by National Institutes of Health (NIH) Grants NS-40296 and MH-104536 to J.T.L. N.A.A.-S. was supported in part by NIH Predoctoral Training Grant T32-GM-007287. We thank Ellie Heckscher and Gerry Rubin for sharing fly lines. We also thank the Bloomington Drosophila Stock Center (Indiana University, Bloomington, IN; NIH Grant P40-0D-018537), the Drosophila Genome Resource Center (Indiana University, Bloomington, IN; NIH Grant 2P40-0D-010949-10A1), the TRiP (enter at Harvard Medical School (Boston, MA; NIH/National Institute of General Medical Sciences Grant R01-GM-084947), the Developmental Studies Hybridoma Bank (University of lowa, lowa City, IA), and the Vienna Drosophila Resource Center (Vienna, Austria) for providing the strains used in this study. In addition, we thank the staff and students in the Cold Spring Harbor Laboratory Drosophila Neurobiology course and members of the Littleton laboratory for sharing resources and advice.

\section{Introduction}

Functional and structural changes in neuronal circuits occur during development and in response to environmental stimuli, learning, and injury (Katz and Shatz, 1996; Destexhe and Marder, 2004; Foeller and Feldman, 2004; Lamprecht and LeDoux, 2004; Holtmaat and Svoboda, 2009). Disruptions of these plasticity pathways contribute to neurodevelopmental

\footnotetext{
Correspondence should be addressed to J. Troy Littleton at troy@mit.edu. https://doi.org/10.1523/JNEUROSCI.0925-20.2020

Copyright $\odot 2020$ Aponte-Santiago et al.

This is an open-access article distributed under the terms of the Creative Commons Attribution License Creative Commons Attribution 4.0 International, which permits unrestricted use, distribution and reproduction in any medium provided that the original work is properly attributed.
} 
A

\begin{tabular}{|l|c|c|}
\hline \multirow{2}{*}{ Morphology } & Ib & Is \\
\cline { 2 - 3 } & Tonic & Phasic \\
\hline Properties & Low $\mathrm{P}_{\mathrm{r}}$ & High $\mathrm{P}_{\mathrm{r}}$ \\
\hline Phobability of release & Facilitation & Depression \\
\hline Synaptic area and SSR & Larger & Smaller \\
\hline Active zone number & Higher & Lower \\
\hline Homeostatic plasticity & Chronic & Acute \\
\hline Spiking Threshold & Lower & Higher \\
\hline $\begin{array}{l}\text { Postsynaptic Target } \\
\text { Innervation }\end{array}$ & $\begin{array}{l}\text { Single } \\
\text { muscle }\end{array}$ & $\begin{array}{l}\text { Multiple } \\
\text { muscles }\end{array}$ \\
\hline
\end{tabular}

\section{C}

MN1-Ib GAL4 Expression

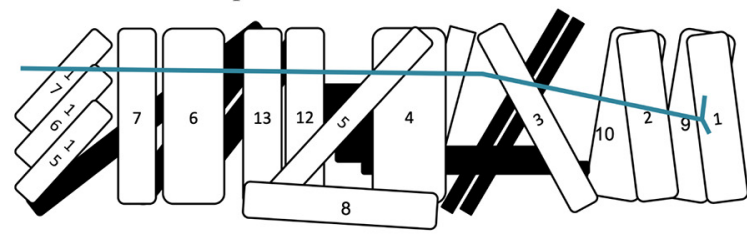

$\mathbf{E}$

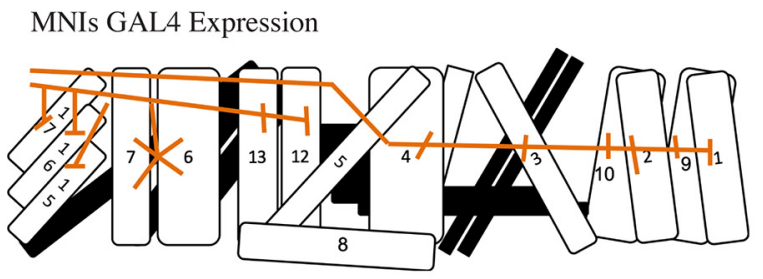

B

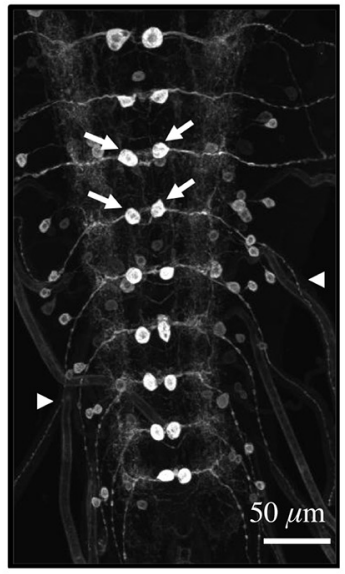

D

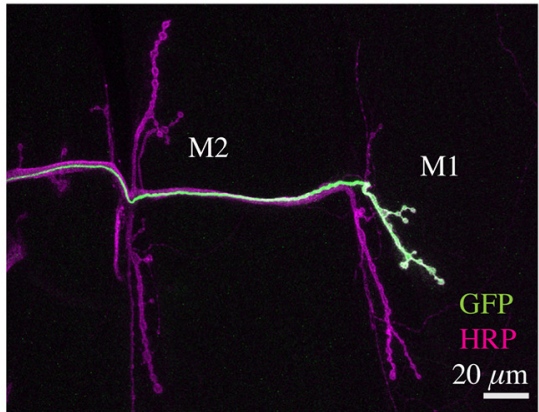

$\mathbf{F}$

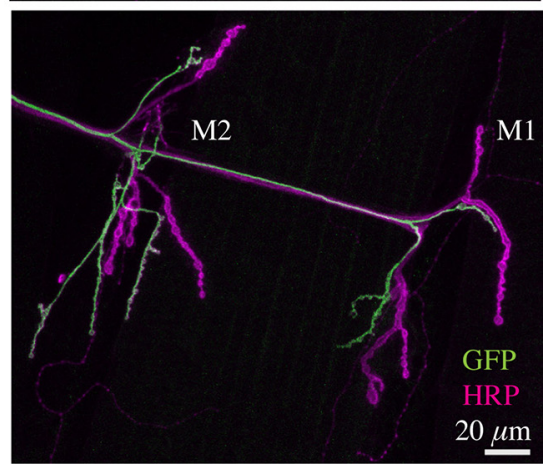

Figure 1. Identification of tonic $\mathrm{Ib}$ and phasic Is motoneuron GAL4 drivers. $\boldsymbol{A}$, Comparison of synaptic and biophysical properties of $\mathrm{Ib}$ and Is motoneurons in Drosophila larvae. $\boldsymbol{B}$, Confocal image of UAS-CD8-GFP driven by MN1-Ib GAL4 (GMR94G06) in the third instar larval VNC from the FlyLight Project GAL4 collection. Arrows denote the paired MN1-lb cell bodies in each abdominal segment, and arrowheads denote GFP expression in axons exiting the VNC. Scale bar, $50 \mu \mathrm{m}$. C, Diagram of MN1-lb innervation in a larval abdominal hemisegment. $\boldsymbol{D}$, Immunostaining for anti-GFP (green) to label MN1-Ib and HRP (magenta) to label all axons in a MN1-Ib GAL4; UAS-CD8-GFP third instar larva. Muscles M1 and M2 are indicated. Scale bar, $20 \mu \mathrm{m}$. $\boldsymbol{E}$, Diagram of MNISN-Is and MNSNb/D-Is innervation in a larval abdominal hemisegment. $\boldsymbol{F}$, Immunostaining for anti-GFP (green) to label MNIs and HRP (magenta) to label all axons in a MNIs GAL4 (6-58); UAS-CD8-GFP third instar larva. Muscles M1 and M2 are indicated. Scale bar, $20 \mu \mathrm{m}$.

diseases and impair rewiring after brain injury, highlighting the importance of the underlying mechanisms (Luo and O'Leary, 2005; Melom and Littleton, 2011; Doll and Broadie, 2014; Nahmani and Turrigiano, 2014). In contrast to mammals, invertebrate nervous systems like that of Drosophila melanogaster are more stereotypical in their organization. Neuroblasts divide and differentiate in a specific order to generate fixed cellular lineages with genetically hardwired synaptic targets (Hartenstein and Campos-Ortega, 1984; Thomas et al., 1984; Johansen et al., 1989a; Bossing et al., 1996; Landgraf et al., 1997; Schmid et al., 1999; Hoang and Chiba, 2001; Yu et al., 2010; Harris et al., 2015; Lee, 2017; Shepherd et al., 2019). Although Drosophila display stereotypical neuronal connectivity, plasticity can occur throughout development and into adulthood. Structural plasticity is most prominent during metamorphosis, when larval neurons reorganize their processes and synaptic partners form functional adult circuits (Technau and Heisenberg, 1982; Truman, 1990; Schubiger et al., 1998; Lee and Luo, 1999; Marin et al., 2005; Williams and Truman, 2005; Alyagor et al., 2018; Mayseless et al., 2018). Alterations in connectivity also occur in response to changes in environmental stimuli or following acute or chronic manipulations of neuronal activity (Cash et al., 1992; Chang and Keshishian, 1996; Davis et al., 1998; Lnenicka et al., 2003; Sigrist et al., 2003; Berdnik et al., 2006; Hourcade et al., 2010; Matz et al., 2010; Golovin et al., 2019).

Although plasticity occurs broadly across neuronal circuits, the motor system has played an important role in defining mechanisms for activity-dependent structural changes in connectivity. Locomotion is an essential behavior in many animals and requires coordinated output from central pattern generators to orchestrate motoneuron $(\mathrm{MN})$ firing patterns that activate specific muscles (Marder and Calabrese, 1996; Marder and Rehm, 2005). In 
vertebrates, individual muscle fibers receive transient innervation from many cholinergic motoneurons during early development (Sanes and Lichtman, 1999). As many as 10 distinct motor axons can innervate a single muscle fiber before an activity-dependent competition results in retention of only a single axon (Tapia et al., 2012). This axonal competition allows a large pool of identical motoneurons to transition from dispersed weak outputs to the muscle field to strong innervation of a smaller subset of muscles (Colman et al., 1997; Walsh and Lichtman, 2003; Turney and Lichtman, 2012).

Unlike vertebrate neuromuscular junctions (NMJs), early promiscuity in synaptic partner choice and subsequent synapse elimination does not occur in Drosophila. Instead, the larval motor system is composed of $\sim 36$ motoneurons from four subclasses that are genetically programmed by specific transcription factors and guidance molecules to form stereotypical connections to the 30 muscles in each abdominal hemisegment (Hoang and Chiba, 2001; Clark et al., 2018). Although synaptic partner choice is hardwired, activity-dependent plasticity and homeostatic mechanisms have been characterized, making Drosophila an ideal system to study synaptic interactions between motor neurons (Davis et al., 1998; Sigrist et al., 2003; Guan et al., 2005; Yoshihara et al., 2005; Frank et al., 2006; Berke et al., 2013; Davis, 2013; Cho et al., 2015; Davis and Müller, 2015; Gaviño et al., 2015; Harris and Littleton, 2015; Böhme et al., 2019; Goel et al., 2019). Although individual muscles normally restrict innervation to a single neuron from each subclass, it is unclear whether motoneurons interact during innervation of the same muscle target or respond when the output of one motoneuron is altered. Therefore, we established a system to differentially manipulate the two primary glutamatergic inputs and characterized the subsequent effects on synaptic morphology and function. We found that only the tonic Ib motoneuron is capable of partially compensating following ablation or silencing of the phasic Is input.

\section{Materials and Methods}

Drosophila stocks. Drosophila melanogaster were cultured on standard medium at $25^{\circ} \mathrm{C}$. Genotypes used in this study include the following: $w^{1118}$ [stock \#3605, Bloomington Drosophila Stock Center (BDSC)]; $13 \times$ LexAop2-mCD8-GFP (stock \#32204, BDSC); UAS-DsRed (stock \#6282, BDSC); UAS-CD8-GFP (stock \#32185, BDSC); UAS-Reaper (stock \#5823, BDSC); UAS-TeTXLC (stock \#28837, BDSC); UASNaChBac (stock \#9469, BDSC); GMR27E09-GAL4 (stock \#49227, BDSC); MN1-Ib-GAL4 (stock \#40701, BDSC); and 6-58 Is-GAL4 (from Ellie Heckscher, University of Chicago, Chicago, IL). Animals of either sex were used depending on the genetic scheme.

Transgenic constructs. To create the MN1-Ib LexA driver line, a 2736 bp fragment from the intronic region of the Dpr4 locus that was used to generate the GMR94G06 MN1-Ib-GAL4 driver line (stock \#40701, BDSC) was PCR amplified and cloned into Invitrogen pCR8/GW/ TOPO (Thermo Fisher Scientific). This was followed by an LR cloning step into the pBPLexA::p65Uw plasmid (catalog \#26231, Addgene). The resulting construct was sent for injection into an attP40 donor site strain by BestGene.

Immunocytochemistry. Third instar wandering larvae were reared at $25^{\circ} \mathrm{C}$ and dissected in hemolymph-like HL3.1 solution as following (in mM): $70 \mathrm{NaCl}, 5 \mathrm{KCl}, 1.5 \mathrm{CaCl}_{2}, 4 \mathrm{MgCl}_{2}, 10 \mathrm{NaHCO}$, 5 trehalose, 115 sucrose, and 5 HEPES, pH 7.2. Larvae were fixed for $10 \mathrm{~min}$ in HL3.1 buffer with $4 \%$ formaldehyde and washed three times for $10 \mathrm{~min}$ with PBT (PBS containing $0.1 \%$ Triton X-100), followed by a 30 min incubation in block solution (5\% NGS in PBT). Fresh block solution and primary antibodies were added. Samples were incubated overnight at $4^{\circ} \mathrm{C}$ and washed with two short washes and three extended 20 min washes in PBT. Secondary antibodies were added to block solution and were incubated at room temperature for $2 \mathrm{~h}$ or at $4^{\circ} \mathrm{C}$ overnight. Finally, larvae
Table 1. GAL4 lines identified in this study

\begin{tabular}{|c|c|c|c|}
\hline Genotype & $\begin{array}{l}\text { GAL4 driver } \\
\text { sequences }\end{array}$ & Protein role & Expression pattern \\
\hline$w^{1118}$;; GMR79HOT-GAL4 & CG3964 & Tubulin tyrosine ligase like & M6 lb (A2 only) \\
\hline$w^{1118}$;; GMR94G06-GAL4 & Dpr4 & Synaptic specificity & MN1-Ib \\
\hline$w^{1118} " ;$ GMR54H01-GAL4 & CG13532 & Ig-like domain superfamily & $\begin{array}{l}\text { MNISN-Is } \\
\text { M1,2,3,4,9,10, } \\
18,19,20 \text { and/ } \\
\text { or MNISN-II } \\
\text { MN12-Ib } \\
\text { MN13-Ib }\end{array}$ \\
\hline$w^{1118} \ddot{\prime \prime G M R 27 F 01-G A L 4 ~}$ & Fmr1 & RNA binding protein & $\begin{array}{l}\text { MNSNb/d-Is, } \\
\text { MNISN-Is, } \\
\text { MNISN-II, }\end{array}$ \\
\hline$w^{1118}$;; GMR25HO8-GAL4 & Milt & Kinesin-associated protein & Type III \\
\hline$w^{1118}, 6-58-G A L 4$ & Dip- $\alpha$ & Synaptic specificity & $\begin{array}{l}\text { MNSNb/d-Is, } \\
\text { MNISN-Is }\end{array}$ \\
\hline
\end{tabular}

The genotype, GAL4 driver enhancer sequence, or site of insertion, predicted protein role, and expression pattern revealed by crossing to UAS-CD8-GFP for third instar larvae is shown for the six lines identified with motoneuron-restricted expression. GMR94G06 and 6-58 are referred to as MN1-lb GAL4 and MNIs GAL4, respectively, throughout the article.

were rewashed and mounted in $80 \%$ glycerol. Antibodies used for this study include the following: mouse anti-BRP (Bruchpilot), 1:500 [stock \#NC82, Developmental Studies Hybridoma Bank (DSHB)]; mouse antiDLG (Discs large), 1:1000 (stock \#4F3, DSHB); chicken anti-DLG, 1:500; DyLight 649 conjugated anti-HRP (horseradish peroxidase), 1:500 (catalog \#123-605-021, Jackson ImmunoResearch); rabbit anti-GFP Alexa Fluor 488, 1:500 (catalog \#G10362, Thermo Fisher Scientific); goat antimouse Alexa Fluor 546, 1:500 (catalog \#A-11030; Thermo Fisher Scientific); and phalloidin-conjugated Alexa Fluor 555 or 657, 1:500 (Thermo Fisher Scientific). Immunoreactive proteins were imaged on a Zeiss Pascal confocal microscope (Carl Zeiss Microscopy) using either a $40 \times 1.3$ numerical aperture (NA), a $63 \times 1.3 \mathrm{NA}$, or a $100 \times 1.3 \mathrm{NA}$ oilimmersion objective (Carl Zeiss Microscopy). Images were processed using Zen software (Zeiss).

Motoneuron GAL4 driver screen. The FlyLight Project image database of larval brain and ventral nerve cord (VNC) GFP expression provided by Gerry Rubin [Janelia Research Campus, Howard Hughes Medical Institute (HHMI)] was searched for GAL4 lines displaying restricted expression in small subsets of segmentally repeated neurons with GFPlabeled axons projecting from the VNC. Candidate lines meeting these criteria were obtained and crossed to UAS-CD8::GFP (stock \#32185, BDSC) for immunostaining with DyLight 649-conjugated anti-HRP (Jackson ImmunoResearch), Alexa Fluor 555 phalloidin (Thermo Fisher Scientific), and rabbit anti-GFP Alexa Fluor 488 (Thermo Fisher Scientific). Confocal imaging was performed to classify labeled neurons based on their synaptic connectivity within the abdominal musculature.

Quantification of confocal images. Imaris 9.2 software (Oxford Instruments) was used to identify BRP puncta to quantify active zone (AZ) number, and HRP labeling was used to quantify the synaptic bouton number from 3D image stacks through the NMJ. For DLG/HRP measurements, the 3D mask feature was used, and the software determined bouton volume within HRP staining and muscle subsynaptic reticulum (SSR) volume from DLG staining. Quantification was conducted at muscle 1 (M1) in abdominal segment A3. The $n$ value refers to the number of NMJs analyzed, with no more than two NMJs analyzed per larvae. Animals used in each analysis were derived from at least three independent experimental crosses. All analysis was performed blind to genotype.

Live imaging of $I b$ and Is innervation and synaptic growth. Live imaging was done under desflurane anesthesia at muscles M1 and M4 at abdominal segments A2-A4 as previously described (Akbergenova et al., 2018). Selected larvae were covered with halocarbon oil and a coverglass and were imaged. After imaging, larvae were placed in numbered chambers with food in a $25^{\circ} \mathrm{C}$ incubator. Larvae were imaged at the beginning of the first instar larval stage and during the subsequent $24 \mathrm{~h}$ interval with the same data acquisition settings. Confocal images were obtained 
A

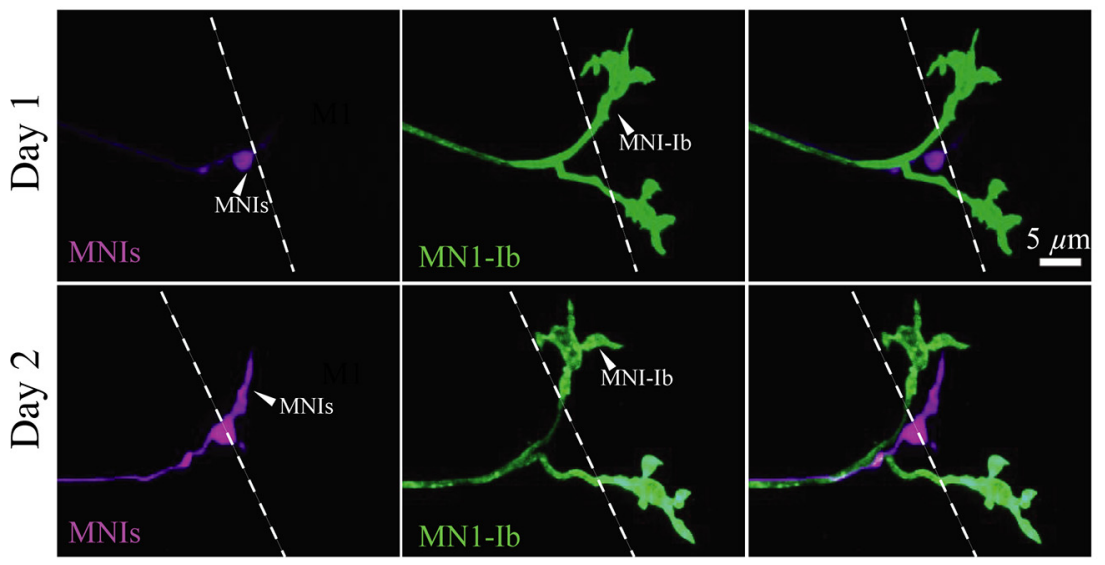

C

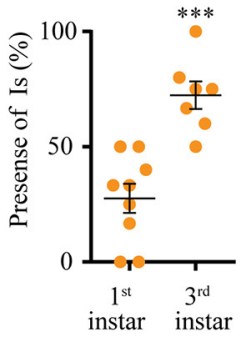

B
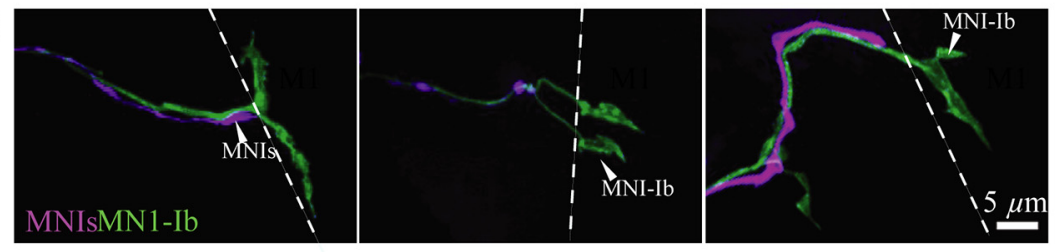

D
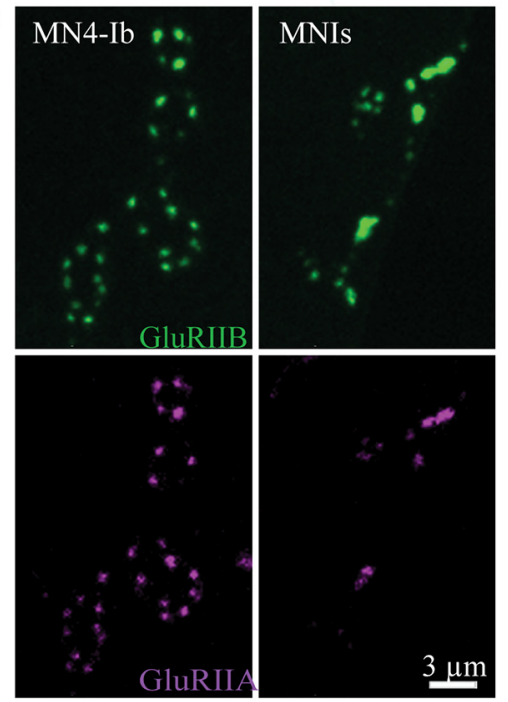

E

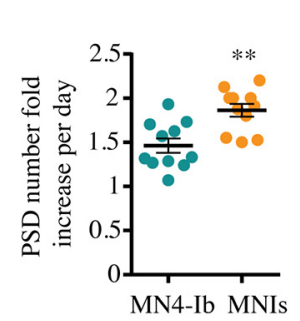

F

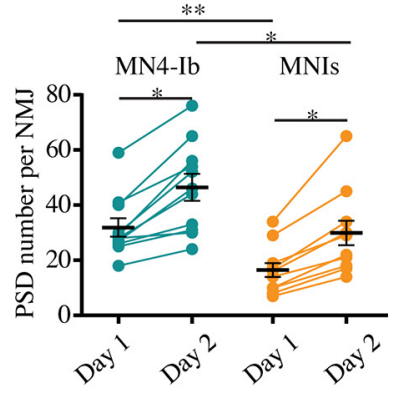

G

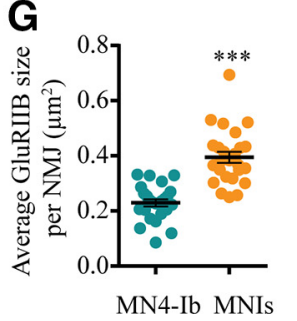

H

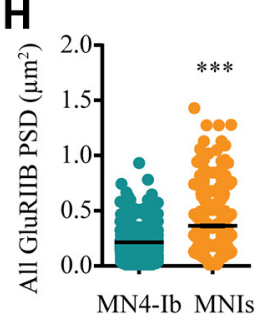

I

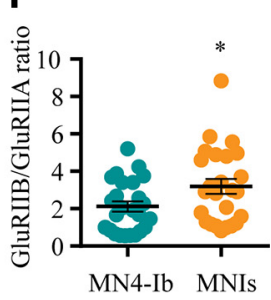

Figure 2. Quantification of MN1-Ib and MNls target innervation and synapse formation with serial intravital imaging across development. $A$, Sequential confocal images of muscle M1 innervation by MN1-Ib (green) and MNIs (magenta) at day 1 (top panels) and day 2 (bottom panels) of larval development in dual-labeled animals (MN1-Ib LexA>LexAop2-CD8-GFP; MNIs GAL4> UAS-DsRed). Dashed line indicates M1 muscle boundary. MNls has delayed innervation compared with MN1-lb. Scale bar, $5 \mu \mathrm{m}$. $\boldsymbol{B}$, Representative confocal images of three M1 muscles on day 1 showing delayed innervation by MNIs (magenta) compared with MN1-Ib (green). MNls axons in the left and middle panels proceeded to innervate M1 later in development, while the MNIs axon on the right failed to innervate M1. Dashed line indicates the M1 muscle boundary. Scale bar, $5 \mu \mathrm{m}$. C, Quantification of Is motoneuron innervation of M1 in first instar (27.6 $\pm 6.3 \%, n=9$ larvae) versus third instar ( $72.4 \pm 6.1 \%, n=7$ larvae; $p=0.0002$, Student's $t$ test). Each point represents the average percentage of M1 innervation in segments A2-A4 from a single larva. $\boldsymbol{D}$, Confocal imaging of PSDs formed at MN4-Ib and MNIs NMJs on M4 in larvae expressing RFP-tagged GluRIIA (magenta) and GFP-tagged GluRIIB (green). Note that the Is terminal has fewer synapses but larger PSDs. Scale bar, $3 \mu \mathrm{m}$. E, Increase in GluRIIB-positive PSDs over $24 \mathrm{~h}$ starting at the first instar larval stage. The increase in PSD number is plotted as the fold-increase of day 2 PSDs over the initial day 1 PSDs for MN4-Ib and MNIs. Each point represents the average increase at M4 from segments A2-A4 for a single larva. $F$, Increase in PSD number at M4 during serial imaging of MN4-lb and MNls over $24 \mathrm{~h}$ beginning at the first instar stage. Each point represents the average PSD number at M4 from segments A2-A4 for a single larva on day 1 and day 2. At the first instar stage, the PSD number at MN4-lb is $31.9 \pm 3.3(n=11)$ and is statistically different $(p=0.0015)$ from PSD number for MNIs $(16.5 \pm 2.6 ; n=11)$. 0n day 2, PSD number at MN4-Ib increases to $46.5 \pm 4.9(n=11)$, and for MNls to $29.9 \pm 4.5(n=11 ; p=0.021)$. Between the 2 consecutive days of imaging, there is a significant growth and addition of new PSDs (MN4-Ib PSD increase, $p=0.023$; MNIs PSD increase, $p=0.017$ ). G, Quantification of GluRIIB-positive PSD area for MN4-Ib and MNIs synapses at M4 in first instar larvae. Each point represents the average PSD area at M4 from segments A2-A4 for a single larva. $\boldsymbol{H}$, Distribution of individual PSD sizes at M4 from segments A2-A4 for MN4-Ib and MNIs NMJs for all first instar larvae imaged ( $n=9$ larvae each). I, Quantification of GluRIIB to GluRlIA ratio at PSDs apposed to MN4-lb or MNIs synapses at M4 in first instar larvae. Each point represents the ratio at M4 from segments A2-A4 for a single larva. Statistical significance was determined using Student's $t$ test. Data are shown as the mean \pm SEM. ${ }^{*} p<0.05$, ${ }^{* *} p<0.01$, ${ }^{* * *} p<0.001$. 
on a Zeiss Axio Imager 2 equipped with a spinning-disk confocal head (model CSU-X1, Yokagawa) and ImagEM X2 EM-CCD camera (Hamamatsu). A pan-APOCHROMAT 63× objective with $1.40 \mathrm{NA}$ from Zeiss (Carl Zeiss Microscopy) was used for imaging.

Electrophysiology. Wandering third instar larvae were dissected in modified HL3.1 saline solution, as follows (in $\mathrm{mm}$ ): $70 \mathrm{NaCl}, 5 \mathrm{KCl}$, $0.3 \mathrm{CaCl}_{2}, 4 \mathrm{MgCl}_{2}, 10 \mathrm{NaHCO}_{3}, 5$ trehalose, 115 sucrose, and 5 HEPES, pH 7.18. For electrophysiology and force recordings, larvae were pinned medial side up at the anterior and posterior ends, an incision was made along the midline, and the visceral organs were removed. All nerves emerging from the CNS were severed at the ventral nerve cord and the $\mathrm{CNS}$, and the ventral nerve cord was removed. Excitatory junctional potentials (EJPs) were elicited by stimulating severed abdominal nerves. A Master 800 A.M.P.I. stimulator (A-M Systems) was used for stimulation via a suction electrode. EJPs were recorded using sharp glass microelectrodes containing a 2:1 mixture of $3 \mathrm{~m}$ potassium chloride $/ 3 \mathrm{M}$ potassium acetate with electrode resistances of 40-80 M . An Axoclamp 2B amplifier (Molecular Devices) was used for signal detection and digitized via a Digidata 1550 digitizer (Molecular Devices). Signals were acquired at $10 \mathrm{kHz}$ using Clampex and were analyzed with Clampfit, MiniAnalysis, and Microsoft Excel. For physiology experiments involving MN1-Ib GAL4, MNIs GAL4, UAS-RPR, MN1-Ib $>$ RPR, MNIs $>$ RPR, UAS-TeTXLC, and MNIs $>$ TeTXLC, the preparations were stained afterward to identify animals that had both Ib and Is innervation at muscle 1. Data were used only in cases where both inputs were present, or in situations where the specific loss of one input was expected (RPR experiments). For $\mathrm{Ib}>\mathrm{NaChBac}$ and $\mathrm{Is}>\mathrm{NaChBac}$ electrophysiology experiments, minimal stimulation protocols were used to verify both inputs were present. Experiments with only Ib input were discarded. All analysis was performed blind to genotype.

Muscle force contraction measurements. Force recordings were obtained using an Aurora Scientific 403A Force Transducer System with a force transducer headstage, amplifier, and digitizer. Larvae were dissected ventral side up in HL3.1 saline containing $1.5 \mathrm{~mm} \mathrm{Ca}^{2+}$. Nerveevoked contractions were generated using stimulation bursts from a Master 8 Stimulator (A. M.P.I.). The duration of single impulses was $5 \mathrm{~ms}$ and interburst duration was kept constant at $15 \mathrm{~s}$. Burst frequency was altered during each individual experiment. Digitized data were acquired using Dynamic Muscle Acquisition Software (DMC version 5.5, Aurora Scientific) and imported and processed in MATLAB using custom code. All analysis was performed blind to genotype.

Statistical analysis. Prism software (version 8.1.1; GraphPad Software), and FIJI/ImageJ software was used for statistical analysis. Statistical significance in two-way comparisons was determined by a Student's $t$ test, while one-way ANOVA parametric analysis was used when comparing more than two datasets. Statistical comparisons are made with control unless noted. Appropriate sample size was determined using a normality test. Data are presented as the mean \pm SEM $\left({ }^{\star} p<0.05,{ }^{\star *} p<0.01,{ }^{* * *} p<0.001\right.$, n.s. $=$ not significant $)$.
A
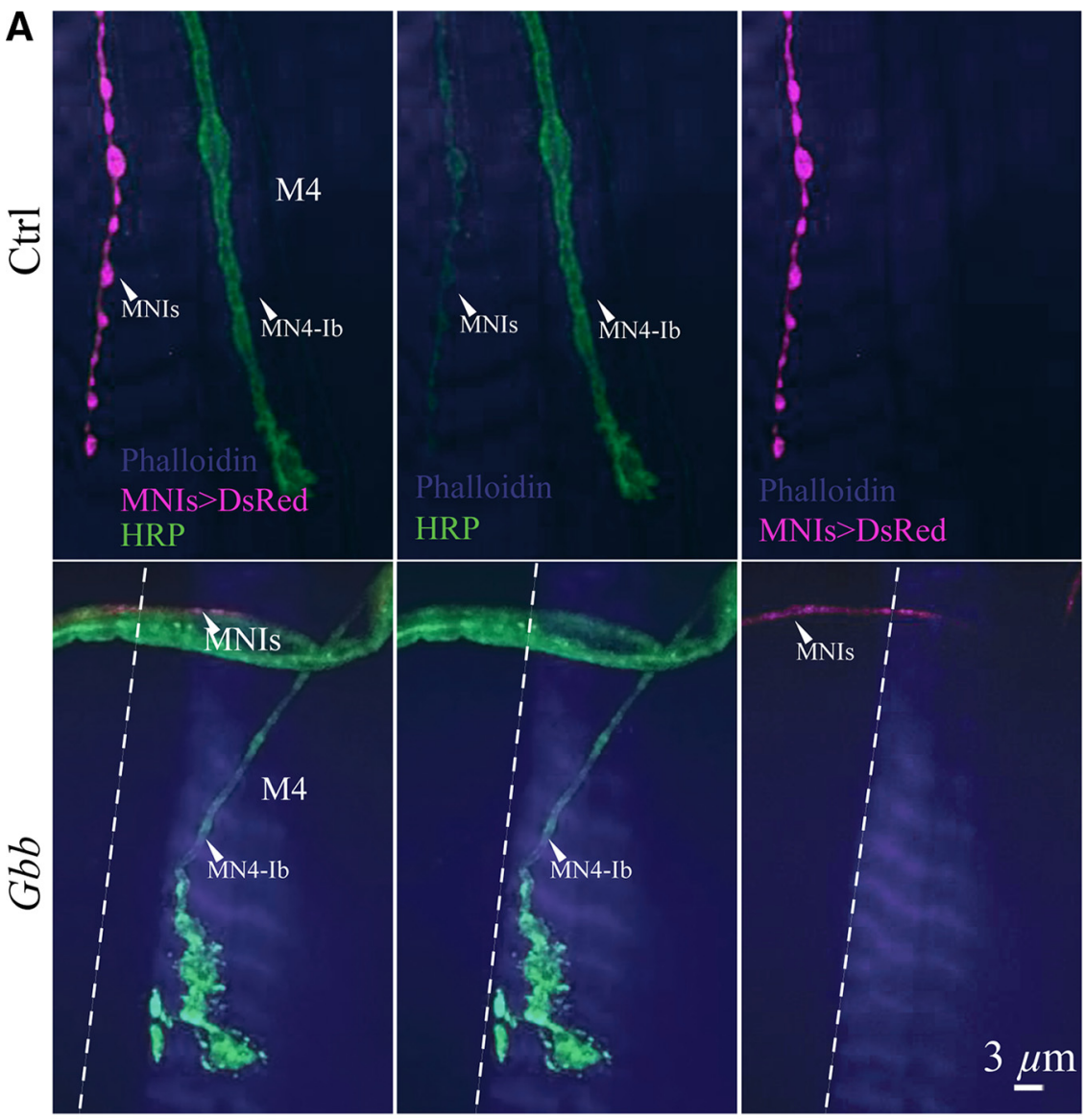

B

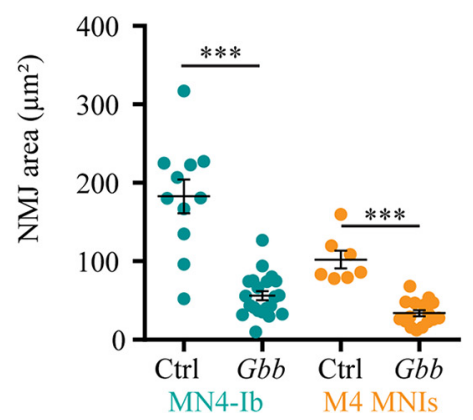

C

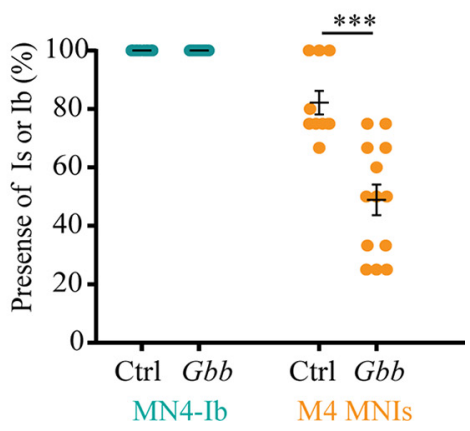

Figure 3. Reduction in synaptic growth and muscle innervation by is motoneurons in Gbb mutants. $\boldsymbol{A}$, Confocal images of third instar muscle M4 innervation by MN4-Ib (green, anti-HRP staining) and MNls (magenta, MNIs GAL4>UAS-DsRed) in controls (top panels) or $G b b$ mutants $\left(g b b^{7} / g b b^{2}\right.$, bottom panels). Muscles were labeled with phalloidin-conjugated Alexa Fluor 647 and are shown in blue. Dashed line indicates M4 muscle boundary and each neuron is labeled. Scale bar, $3 \mu \mathrm{m}$. $\boldsymbol{B}$, Each point represents the average NMJ area at M4 from segments A2-A4 for a single larva. C, Quantification of the percentage of M4 muscles innervated by MN4-Ib or MNIs at the third instar stage. Each point represents the average percentage of M4 innervation in segments A2-A4 from a single larva. Statistical significance was determined using Student's $t$ test. Data are shown as the mean \pm SEM. ${ }^{* * *} p<0.001$.

\section{Results}

\section{Screen for Ib and Is motoneuron-specific GAL4 drivers}

Four subclasses of motoneurons innervate the abdominal musculature in Drosophila, with each class defined by their synaptic partner choice, neurotransmitter or neuromodulator content, and biophysical and synaptic properties (Jan and Jan, 1976; Johansen et al., 1989a; Atwood et al., 1993; Lnenicka and Keshishian, 2000; Hoang and Chiba, 2001). Approximately 30 type $\mathrm{Ib}$ glutamatergic motoneurons are found per hemisegment and function as the primary driver of contraction for individual muscles. A single Ib motoneuron individually innervates a single 
A
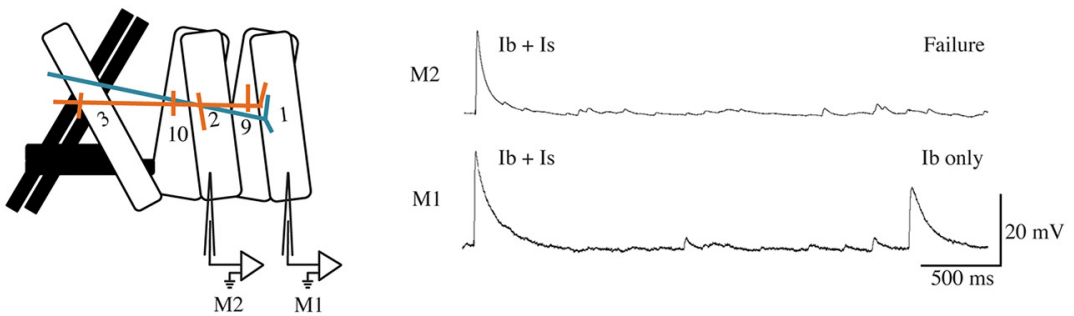

B

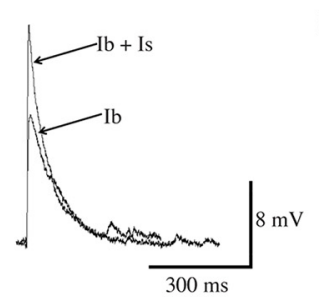

C

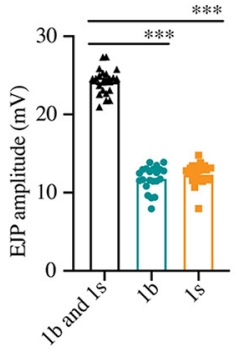

D

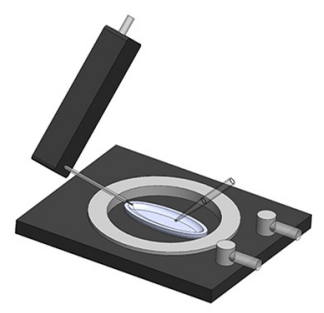

E

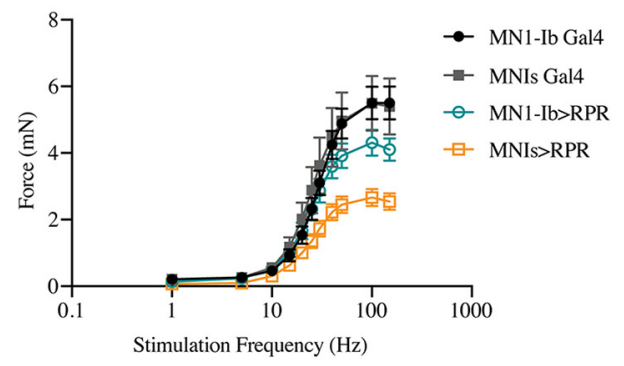

G

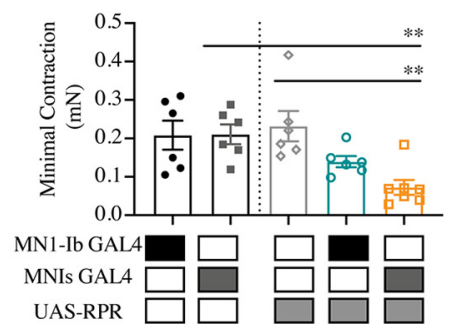

Figure 4. Contributions of MN1-lb and MNIs to muscle excitability and contractile force. $A$, Depiction of a dual intracellular electrode paradigm for performing simultaneous voltage recordings from muscles M1 and M2 in control $w^{178}$ third instar larvae, with MN1-Ib (teal) and MNIs (orange) labeled. Representative recordings from M1 and M2 are shown on the right. Ib + Is shows the compound EJP generated when both motoneurons are activated. Lowering stimulation intensity results in the recruitment of only MN1-lb or MNIs. Stimulation of only MNls triggers responses in both muscles, given that it innervates M1 and M2. Stimulation of MN1-lb, as shown in the lb-only trace, results in responses only from M1. $\boldsymbol{B}$, Representative traces of simple or compound EJPs at M1 showing recruitment of MN1-Ib only or both MN1-lb and MNIs. C, Average EJP amplitude at M1 following the recruitment of both motoneurons or MN1-lb or MNls only ( $n=22$ larvae). $\boldsymbol{D}$, Schematic of force transducer setup used to measure larval muscle contractile force. $\boldsymbol{E}$, Force-frequency curves for $1-150 \mathrm{~Hz}$ nerve stimulation in $\mathrm{MN1}-\mathrm{Ib}$ and MNIs GAL4 controls and MN1-Ib GAL4 $>$ RPR and MNIs GAL4 $>$ RPR ablated third instar larvae. Six replicate contractions were generated at each stimulation frequency for each recording and averaged across seven larvae per genotype. $\boldsymbol{F}$, Maximal contraction force elicited at $150 \mathrm{~Hz}$ is shown. Shaded boxes under each bar indicate the genotypes for each experimental group. $\mathbf{G}$, Minimal contraction force elicited by a single action potential for the indicated genotypes. Statistical significance was determined using Student's $t$ test. Data are shown as the mean \pm SEM. ${ }^{* *} p<0.01,{ }^{* * *} p<0.001$.

muscle fiber, allowing for fine-tuning of specific locomotor programs. The Ib neuronal subclass has big synaptic boutons containing AZs with low release probability $\left(P_{r}\right)$ that facilitate during high-frequency stimulation (Lnenicka and Keshishian, 2000; Peled and Isacoff, 2011; Melom et al., 2013; Newman et al., 2017; Akbergenova et al., 2018). We refer to the Ib subtype as tonic to denote the sustained firing during muscle contractions cycles, low release probability, and short-term facilitation. Three type Is glutamatergic motoneurons per hemisegment provide input to the ventral, lateral, or dorsal muscle groups, respectively. Each Is neuron innervates multiple fibers to coordinate the contraction of functionally related muscles. In contrast to Ib motoneurons, Is motoneurons have smaller synaptic boutons and fewer AZs with higher $P_{r}$ release sites that undergo depression during repetitive stimulation (Fig. $1 A$ ). We refer to the Is subtype as phasic to denote that it has stronger synapses that rapidly undergo depression, with a firing pattern that drives rapid coactivation of specific muscle groups. The remaining type II and III subclasses are neuromodulatory in nature (Gorczyca et al., 1993; Stocker et al., 2018).

To preferentially manipulate $\mathrm{Ib}$ and Is motoneurons, GAL4 lines with subclass-specific expression were identified from the FlyLight Project (Jenett et al., 2012; Manning et al., 2012). and strains were provided by Ellie Heckscher (University of Chicago). The FlyLight collection consists of $>5000$ transgenic Drosophila lines with $\sim 3 \mathrm{~kb}$ of regulatory genomic DNA from candidate neuronal genes driving GAL4 expression. Images of membrane-tethered UASCD8-GFP driven by each GAL4 line in third instar brain lobes and VNC were provided by Gerry Rubin (Janelia Research Campus, HHMI). Candidate lines for further analysis were chosen on the basis of two criteria: (1) restricted expression of GFP in a single pair or a small subset of segmentally repeated abdominal neurons in the VNC; and (2) GFP expression in axons exiting the VNC as expected for motoneurons innervating peripheral musculature (Fig. 1B). Forty-two GAL4 driver lines were identified as promising candidates in the initial screen and subjected to further immunostaining to identify their synaptic targets. Six of these lines were verified as having restricted expression in a small subset of motoneurons (Table 1), including GAL4 drivers specific for $\mathrm{Ib}$ and Is motoneurons (Fig. $1 C-F$ ). Line GMR94G06 displayed restricted expression in the Ib motoneuron (MN1-Ib) that innervates muscle M1 (Fig. $1 B-D$ ). GMR94G06 contains regulatory DNA from the Dpr4 locus, which encodes a member of the cell surface Ig-containing proteins implicated in synaptic target recognition (Carrillo et al., 2015). Line GMR27F01 contained regulatory sequences from the Fmrl gene and showed restricted expression in two Is motoneurons and a type II neuromodulatory neuron (Table 1). Line 6-58 displayed restricted expression in the Is motoneurons MNISN-Is and MNSNb/D-Is that innervate the ventral and dorsal muscles, respectively (Fig. $1 E, F$ ). To determine the gene regulatory region responsible for Is motoneuron expression in 658 , which contained an unknown insertion site, plasmid rescue and reverse PCR was performed. The insertion site mapped to 
the $5^{\prime}$ UTR of the Dip- $\alpha$ gene. Like DPR4, DIP- $\alpha$ is a member of the Ig domain family of synaptic target recognition proteins and was previously found to be expressed in Is motoneurons (Ashley et al., 2019). The MN1-Ib driver GMR94G06 and an additional Is GAL4 driver line (GMR27E09, containing regulatory sequences from $\mathrm{Fmr} 1$ ) were independently identified in a recent study (Pérez-Moreno and O'Kane, 2019). We refer to the restricted GAL4 driver lines as MN1-Ib GAL4 and MNIs GAL4 in the remaining text. Together, they provide a toolkit to genetically manipulate the tonic Ib and phasic Is motoneuron subclasses that coinnervate muscle M1.

\section{Establishment of MN1-Ib and MN-Is synaptic connections during development} MN1-Ib and MNIs drivers were used to fluorescently label the two neuronal subpopulations and establish the timing for M1 innervation, the most peripheral target of the dorsal abdominal musculature. MN1-Ib (formerly referred to as aCC) and MNIs (formerly referred to as RP2) were previously identified as pioneer neurons for the ISN nerve branch, being the first to exit the VNC toward the dorsal muscle field (Jacobs and Goodman, 1989; Johansen et al., 1989b; Lin et al., 1995; Sánchez-Soriano and Prokop, 2005). To colabel MN1-Ib and MNIs in the same animal, an MN1-Ib LexA driver line was generated by subcloning the 2736 bp genomic Dpr4 fragment from GMR94G06 into pBPLexA::p65Uw. This construct was used to generate transgenic animals containing MN1-Ib LexA, allowing independent LexA and GAL4 transgene expression in MN1-Ib and MNIs motoneurons. Serial intravital imaging through the cuticle of briefly anesthetized animals coexpressing MN1-Ib Lex; LexAop2-CD8-GFP and MNIs GAL4; UAS-DsRed was performed as previously described (Akbergenova et al., 2018). By the beginning of the first instar larval stage, all MN1-Ib motoneurons had correctly targeted M1 during late embryonic development, elaborating a growth cone-like projection over the muscle surface (Fig. 2A,B). In contrast, MNIs had a more variable time course of innervation, with the Is growth cone trailing behind MN1-Ib, often without targeting M1 in early first instars. By the end of the first instar larval stage, only $28 \%$ of MNIs motoneurons had innervated the muscle. MNIs innervation of M1 continued over the rest of larval development, with $72 \%$ of Is motoneurons innervating $\mathrm{M} 1$ by the third instar stage (abdominal segments 24; $n=7$ larvae; Fig. 2C). The remaining M1 muscles lacked Is innervation. These data indicate that the $\mathrm{Ib}$ motoneuron innervates $\mathrm{M} 1$ before the arrival of Is, with the Is motoneuron forming synaptic contacts with the muscle later in development or failing to innervate the target completely.

Since phasic Is motoneurons have stronger synapses with higher $P_{r}$ active zones than their tonic Ib counterparts at the third instar stage (Kurdyak et al., 1994; Lnenicka and Keshishian,
B
M1

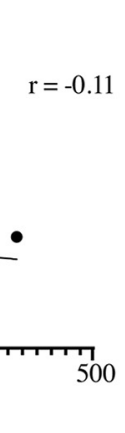

D

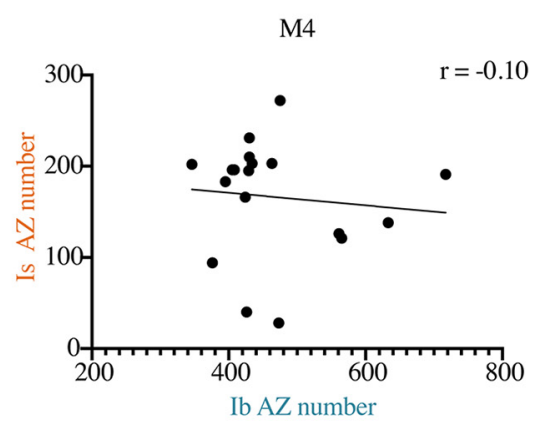

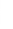

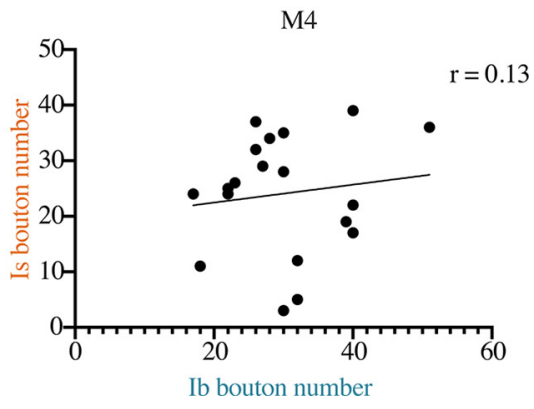

Figure 5. Lack of correlation between Ib and Is synaptic innervation at M1 and M4. A, Correlation of MN1-lb and MNIs AZ $p=0.60$ ). The Pearson correlation coefficient $(r)$ is shown on the top right for each analysis. Each data point corresponds to $\mathrm{Ib}$ and Is AZ or bouton number from a single larva at the indicated muscle of segment A3.

2000; Lu et al., 2016; Newman et al., 2017; Genç and Davis, 2019; Karunanithi et al., 2020), we examined Is synaptic maturation given their shorter developmental time window compared with the pre-existing Ib input. Presynaptic bouton and AZ number at motoneuron NMJs increase throughout larval development to help maintain normal levels of depolarization during the rapid expansion of muscle size during this period. This synaptic growth is accompanied by expansion in the size of the postsynaptic density (PSD) and glutamate receptor fields (Zito et al., 1999; Akbergenova et al., 2018). To examine glutamate receptor field formation and maturation at developing Ib and Is synapses, we followed M4 innervation during early larval development (Fig. 2D). The Ib and Is inputs arrive at distinct positions on M4, allowing unambiguous identification of neuronal subclass without having to genetically label the motoneurons, as required for M1. Live imaging was performed in developing larvae expressing RFP-tagged GluRIIA and GFP-tagged GluRIIB under the control of their endogenous promotors (Rasse et al., 2005). Glutamate receptors at the NMJ are tetramers, with three essential subunits and a fourth subunit of either GluRIIA or GluRIIB (Schuster et al., 1991; Petersen et al., 1997; Marrus et al., 2004; Featherstone et al., 2005; Qin et al., 2005). As observed at M1, MN4-Ib innervation preceded MNIs arrival at M4, with $18 \%$ of M4 fibers completely lacking Is innervation at the third instar stage (abdominal segments A2-A4, $n=9$ larvae). Although Is innervation of M4 was delayed compared with $\mathrm{Ib}$, the fractional rate of synapse addition was elevated at Is terminals during consecutive days of imaging as quantified by the appearance of new GluRIIA/ GluRIIB-positive PSDs (MN4-Ib, 1.46-fold increase; MNIs, 1.86- 
fold increase; $n=11, p=0.0014$; Fig. $2 E, F)$. Overall, the delayed innervation by Is resulted in a reduced PSD number at M4 compared with Ib (day 1: MN4-Ib, 31.9 \pm 3.3 AZs; MNIs, $16.5 \pm 2.6$ AZs; day 2: MN4-Ib, 46.5 \pm 4.9 AZs; MNIs, $29.9 \pm 4.5$ AZs; $n=11, p=0.0014$; Fig. $2 F)$. Although the Is motoneuron formed fewer synapses than $\mathrm{Ib}$, the average PSD size defined by the GluRIIB area in first instar larvae was $69 \%$ larger than those of the corresponding Ib input (MN4-Ib: $0.215 \pm 0.004 \mu \mathrm{m}^{2}, n=$ 1006 PSDs; MNIs: $0.363 \pm 0.013 \mu \mathrm{m}^{2}, n=399$ PSDs; $p=1.2 \times$ $10^{-8}$; Fig. $\left.2 G, H\right)$. Given that PSD maturation is activity dependent at NMJs (Schmid et al., 2008; Petzoldt et al., 2014; Akbergenova et al., 2018), these data suggest that PSDs may develop faster at the stronger Is AZs than PSDs apposed to weaker Ib AZs. Alternatively, the postsynaptic muscle may compartmentalize the delivery of PSD material to Is versus Ib synapses such that Is sites are favored for the accumulation of glutamate receptors incorporating the IIB subunit.

To further characterize the differences in postsynaptic receptor composition between MN4-Ib and MNIs, GluRIIA PSD levels were assayed to determine whether they were also proportionally increased when apposed to MNIs AZs. No difference in GluRIIA PSD accumulation at MN4-Ib versus MNIs first instar synapses was found (MN4-Ib: $6268 \pm 881.9$ GluRIIA fluorescent intensity units, $n=25$; Is: $7767 \pm 1040, n=25$; $p=0.73$ ). A key feature of Drosophila PSD maturation is a change in the ratio of GluRIIB and GluRIIA subunit-containing receptors during development (Schmid et al., 2008). Comparative analysis of the GluRIIB/GluRIIA ratio revealed that it was shifted in favor of the GluRIIB subunit at PSDs apposed to MNIs AZs (3.19 \pm 0.4 ratio, $n=25)$ versus MN4-Ib AZs $(2.12 \pm 0.27$ ratio, $n=25 ; p=0.03$; Fig. 2I). These data suggest that different synaptic inputs innervating the same muscle can display distinct PSD glutamate receptor subunit incorporation. Overall, we conclude that PSDs form at a slightly elevated rate at MNIs connections during this early growth window and that they preferentially accumulate the GluRIIB subunit versus the GluRIIA variant.

A central pathway regulating synaptic maturation at Drosophila NMJs is mediated through muscle secretion of Glass Bottom Boat (Gbb), a bone morphogenetic protein (BMP) ligand that acts on presynaptic BMP receptors to activate a SMAD-dependent transcriptional synaptic growth program (Aberle et al., 2002; Marqués et al., 2002; McCabe et al., 2003; Ball et al., 2010; Rodal et al., 2011; Berke et al., 2013). To determine whether Ib and Is motoneurons were equally sensitive to Gbb signaling given their different innervation time course, we assayed synapse formation and growth of MN4-Ib and MNIs at M4 in Gbb mutants (Fig. 3A). As previously observed, loss of Gbb reduced the synaptic growth of Ib motoneurons innervating M4 compared with controls (control MN4-Ib NMJ area: $182.8 \pm 21.6 \mu \mathrm{m}^{2}, n=11$ NMJs from 5 larvae; $G b b$ MN4-Ib NMJ area: $56.2 \pm 5.8 \mu^{2}, n=21$ NMJs from 8 larvae; $p=0.0001$; Fig. $3 B$ ). Although synaptic growth was reduced, $100 \%$ of M4 muscles displayed Ib synaptic innervation. In contrast, synaptic innervation from the Is motoneuron was reduced in $G b b$ mutants, with only $48 \%$ of M4 muscles containing Is innervation compared with $82 \%$ in controls (Fig. $3 C$ ). In cases where the Is motoneuron innervated M4 in $G b b$, similar reductions in synaptic growth compared with Ib were observed (control Is NMJ area: $102.2 \pm 11.3 \mu \mathrm{m}^{2}, n=7 \mathrm{NMJ}$ s from 5 larvae; Gbb Is NMJ area: $33.8 \pm 4.0 \mu \mathrm{m}^{2}, n=16$ NMJs from 8 larvae; $p=0.0006$; Fig. $3 B$ ). These data indicate the Gbb pathway promotes synaptic growth in tonic $\mathrm{Ib}$ motoneurons but is not required for target innervation. In contrast, the loss of Gbb signaling in phasic Is motoneurons
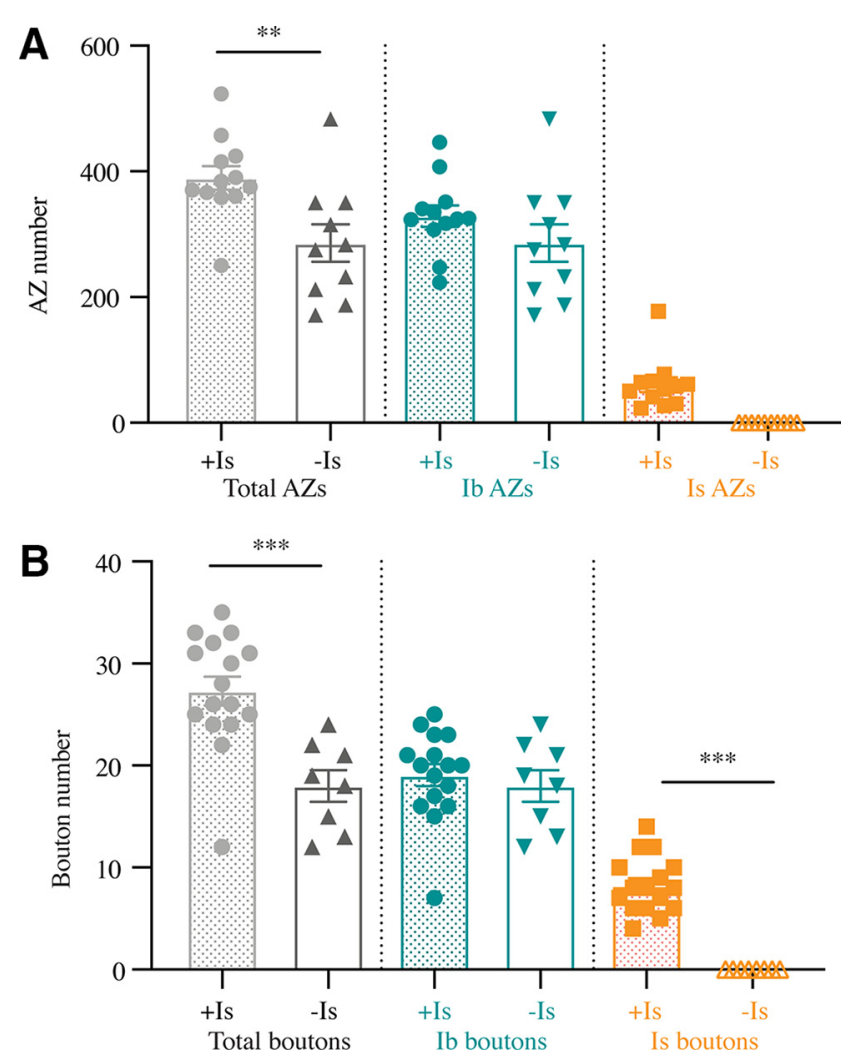

Figure 6. Lack of structural synaptic changes in MN1-lb when Is innervation is absent. $\boldsymbol{A}$, Quantification of MN1-lb and MNIs AZ number following immunolabeling for BRP in control $w^{118}$ third instar larval M1 muscles in segment A3. The total AZ number when both inputs are present $(+\mid \mathrm{Is})$ or when Is innervation is absent $(-\mathrm{Is})$ is shown. AZ number specifically for MN1-Ib (teal) or MNIs (orange) is also shown when both inputs are present $(+\mid \mathrm{I})$ or when Is innervation is absent (-Is). $\boldsymbol{B}$, Quantification of MN1-Ib and MNIs synaptic bouton number following immunolabeling for HRP in $w^{118}$ third instar larval M1 muscles in segment A3. Total bouton number when both inputs are present $(+\mathrm{Is})$ or when Is innervation is absent (-Is) is shown. Bouton number specifically for MN1-lb (teal) or MNIs (orange) is also shown when both inputs are present $(+\mid \mathrm{I})$ or when Is innervation is absent $(-I s)$. Each data point represents quantification from a single larva. Statistical significance was determined using ANOVA. Data are shown as the mean \pm SEM. ${ }^{* *} p<0.01,{ }^{* * *} p<0.001$.

decreases synaptic growth and also reduces the percentage of muscles with Is innervation.

\section{Role of MN1-Ib and MNIs in muscle excitability and contraction}

To determine the relative contributions of $\mathrm{Ib}$ and Is motoneurons in muscle excitability, simultaneous electrophysiological recordings were performed at third instar larval muscles M1 and M2 in HL3.1 saline solution containing $0.3 \mathrm{~mm}$ extracellular $\mathrm{Ca}^{2+}$. A minimal stimulation protocol was used to isolate MN1Ib or MNIs, as MNIs innervates both muscles compared with MN1-Ib (Fig. 4A). By increasing the current applied to the larval nerve through the stimulating electrode, responses following the activation of one or both motor axons could be isolated in cases where dual innervation was present. The average EJP amplitude recorded at $\mathrm{M} 1$ when both $\mathrm{Ib}$ and Is inputs were active was $24.2 \pm 1.7 \mathrm{mV}(n=22$; Fig. $4 B, C)$. When Ib or Is was individually recruited during minimal stimulation, reduced responses of similar amplitude at M1 were observed (MN1-Ib: $11.8 \pm 1.6 \mathrm{mV}$, $n=22$; MNIs: $12.4 \pm 1.4 \mathrm{mV}, n=22$ ), indicating that each neuron provides similar drive to $\mathrm{M} 1$ following single action potentials (Fig. 4B,C). Given that MN1-Ib has more AZs compared with MNIs at M1 (Fig. 2F), these results are consistent with MNIs 
motoneurons having higher $P_{r}$ per $\mathrm{AZ}$ as previously described (Lu et al., 2016; Newman et al., 2017; Genç and Davis, 2019).

To examine the contribution of MN1Ib and MNIs for muscle contractility, nerve-evoked bodywall contraction force was recorded with a force transducer attached to the head of dissected larvae (Fig. 4D). To isolate contraction force mediated predominantly by the dorsal muscle group (M1, M2, M3, M9, and M10), third instar larvae were dissected along the ventral midline. Severed abdominal nerves were placed in a suction electrode, and the nerve bundle was stimulated at increasing frequencies in HL3.1 saline containing $1.5 \mathrm{~mm}$ extracellular $\mathrm{Ca}^{2+}$ as previously described (Ormerod et al., 2016). Control MN1-Ib and MNIs GAL4 larval preparations showed increasing contraction force when a $0.1 \mathrm{~ms}$ simulation was ramped from 1 to $150 \mathrm{~Hz}$ for $600 \mathrm{~ms}$ (Fig. 4E). To determine the contribution of each motoneuron subclass for contractile force, MN1-Ib or MNIs was ablated by expressing the cell death gene reaper (UAS-RPR) to induce apoptosis (White et al., 1994, 1996; Goyal et al., 2000). Expression of UAS-RPR with MN1-Ib or MNIs GAL4 resulted in elimination of the corresponding motoneuron class. Ablation of MNIs removed phasic input to all dorsal and ventral muscles and resulted in a robust reduction in contractile force over the entire frequency distribution, including a 53\% decrease in maximum force following $150 \mathrm{~Hz}$ stimulation (MNIs GAL4: $5.4 \pm 0.2 \mathrm{mN}$, $n=7$; MNIs > UAS-RPR: $2.5 \pm 0.1 \mathrm{mN}$, $n=8 ; p=0.009$; Fig. $4 F)$ and a $65 \%$ decrease in minimal contractile force following a single action potential (MNIs GAL4: $0.2 \pm 0.02 \mathrm{mN}, n=7$; MNIs $>$ RPR: $0.07 \pm 0.02 \mathrm{mN}, n=7 ; p=0.0009$; Fig. $4 G$ ). Ablation of MN1-Ib eliminated tonic input only to M1, leaving innervation of the other dorsal muscles by their respective Ib and Is neurons intact. Loss of MN1-Ib caused a less severe defect, resulting in a $25 \%$ decrease in contractile force at $150 \mathrm{~Hz}$ (MN1-Ib GAL4: $5.5 \pm 0.2 \mathrm{mN}, n=7$; MN1-Ib>UAS-RPR: $4.1 \pm 0.2 \mathrm{mN}, n=7 ; p=0.022$; Fig. $4 F)$ and a $33 \%$ decrease at $1 \mathrm{~Hz}$ (MN1-Ib GAL4: $0.21 \pm 0.02 \mathrm{mN}, n=7$; MN1-Ib>UAS-RPR: $0.14 \pm 0.01 \mathrm{mN}, n=7 ; p=0.068$; Fig. $4 G$ ). Although it is difficult to directly compare the contributions of Ib versus Is ablation in force contraction assays given that Is innervates multiple muscles, these findings suggest that both motoneuron subclasses contribute to muscle contraction force, with the phasic Is input providing the drive for both excitability and contraction.

\section{Lack of Ib and Is synaptic competition during NMJ development}

Given the role of $\mathrm{Ib}$ and Is motoneuron inputs in driving muscle excitability, we examined whether interactions between the inputs occurred during larval development that shaped their axonal arbor expansion and AZ number when they coinnervated M1 or M4. If Ib and Is neurons competed for synaptic growth signals emanating from the muscle, or suppressed the growth of the coinnervating input, competitive interactions should generate a negative correlation between $\mathrm{Ib}$ and Is synapse number. If the two inputs display cooperative interactions during growth, for example by coactivating the muscle to release more synaptogenic factors, one would expect a positive correlation. Similarly, if both inputs were independent and growing only in response to muscle size, a positive correlation would be expected. Ib and Is synaptic terminals were identified following anti-DLG immunostaining. DLG is a component of the postsynaptic muscle SSR and is prominent around presynaptic Ib boutons compared with Is (Lahey et al., 1994). Synapse number was quantified for wandering third instar larvae by immunolabeling for the core AZ T-bar component BRP (Wagh et al., 2006; 

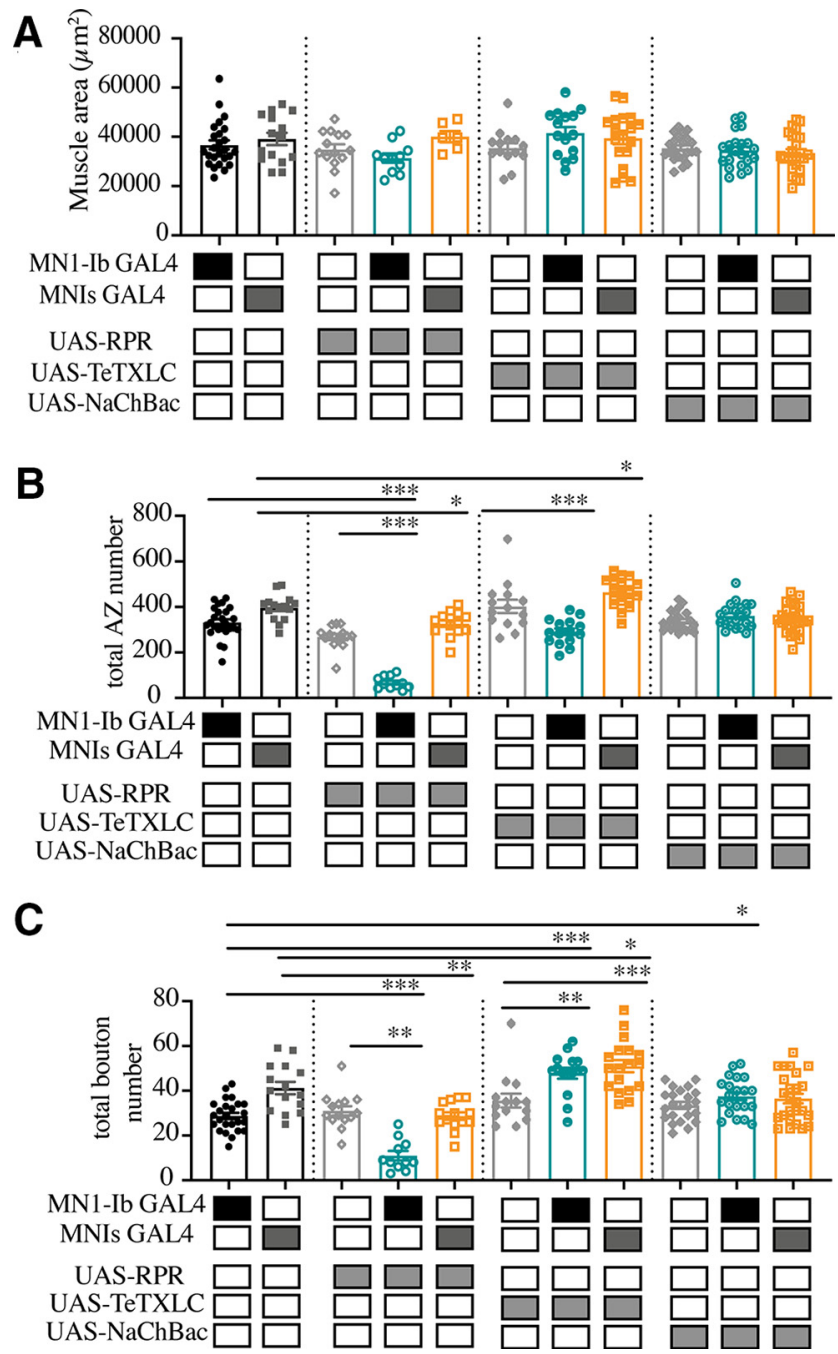

Figure 8. Quantification of muscle size, the total AZ and total bouton number follow ablation, or activity changes of MN1-lb or MNIs is shown. $A$, M1 muscle size is not altered by ablation or activity changes of MN1-Ib or MNIs motoneurons. Shaded boxes under each bar indicate the genotypes for each group, with control GAL4 driver lines alone (MN1-Ib, MNIs), control UAS transgenes alone (UAS-RPR, UAS-TeTXLC, UAS-NaChBac), and experimental crosses of MN1-Ib GAL4 (teal) or MNIs GAL4 (orange) to each transgene. Each data point represents quantification of segment $\mathrm{A} 3 \mathrm{M} 1$ surface area from a single third instar larvae. No statistical difference was found across genotypes. B, Quantification of combined MN1-lb and MNIs AZ number following immunolabeling for BRP in third instar larval M1 muscles in segment A3 of the indicated genotypes. C, Quantification of combined MN1-lb and MNIs synaptic bouton number following immunolabeling for HRP in third instar larval M1 muscles in segment $\mathrm{A} 3$ of the indicated genotypes. Shaded boxes under each bar indicate the genotypes for each group, with control GAL4 driver lines alone (MN1-Ib, MNIs), control UAS transgenes alone (UAS-RPR, UAS-TeTXLC, UAS-NaChBac), and experimental crosses of MN1-lb GAL4 (teal) or MNIs GAL4 (orange) to each transgene. Each data point represents quantification from segment $\mathrm{A} 3 \mathrm{M} 1$ from a single third instar larvae. Statistical significance was determined using ANOVA. Data are shown as the mean \pm SEM. ${ }^{*} p<0.05,{ }^{* *} p<0.01,{ }^{* * *} p<0.001$.

Fouquet et al., 2009). Synaptic bouton number was determined using anti-HRP immunostaining, which provides a neuron-specific membrane label. No correlation was observed between $\mathrm{Ib}$ and Is AZ number per NMJ at M1 $(r=-0.11, n=29, p=0.57$, Pearson $R$; Fig. $5 A$ ) or between Ib and Is inputs at M4 (Pearson $R=-0.10, n=19, p=0.69$; Fig. $5 B)$. Similarly, no correlation for Ib versus Is bouton number at M1 (Pearson $R=0.15, n=29$, $p=0.44$; Fig. $5 C$ ) or M4 (Pearson $R=0.13, n=19, p=0.60$; Fig. $5 D$ ) was found. We also quantified synaptic interactions between MN4-Ib and MNIs at M4 in Gbb mutants. Although the absence
A
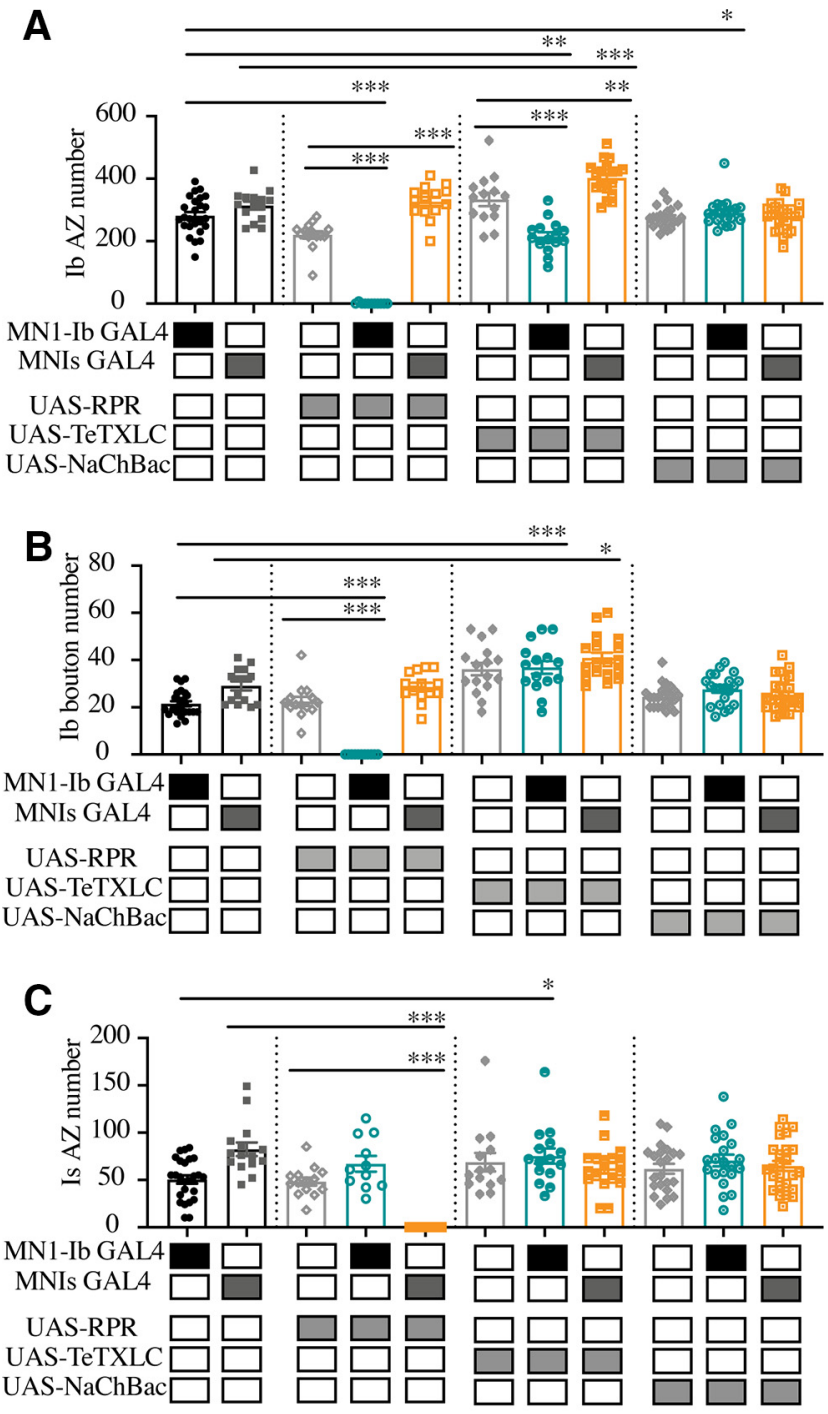

D

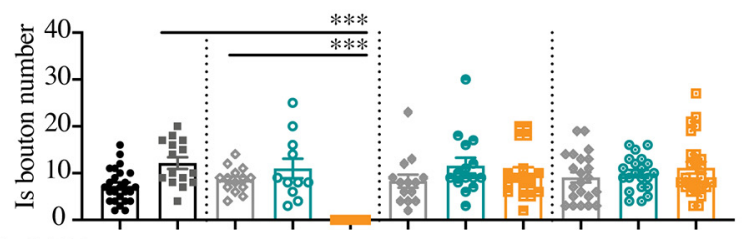

MN1-Ib GAL4 घ $\square \square \square \square \square \square \square \square$

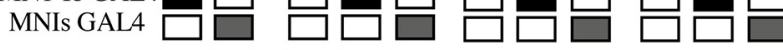
UAS-RPR $\square \square \square \square \square \square \square \square \square \square \square$

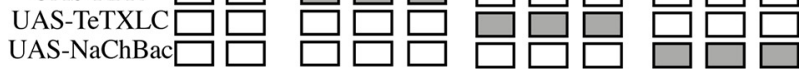

Figure 9. Quantification of MN1-Ib or MNIs AZ and bouton number follow ablation or activity changes. $A$, Quantification of MN1-lb AZ number following immunolabeling for BRP in third instar larval M1 muscles in segment A3 of the indicated genotypes. $\boldsymbol{B}$, Quantification of combined MN1-lb synaptic bouton number following immunolabeling for HRP in third instar larval M1 muscles in segment A3 of the indicated genotypes. C, Quantification of MNIs AZ number following immunolabeling for BRP in third instar larval M1 muscles in segment A3 of the indicated genotypes. $\boldsymbol{D}$, Quantification of MNIs synaptic bouton number following immunolabeling for HRP in third instar larval M1 muscles in segment A3 of the indicated genotypes. Shaded boxes under each bar indicate the genotypes for each group, with control GAL4 driver lines alone (MN1-lb, MNIs), control UAS transgenes alone (UAS-RPR, UASTeTXLC, UAS-NaChBac), and experimental crosses of MN1-Ib GAL4 (teal) or MNIs GAL4 (orange) to each transgene. Each data point represents quantification from segment A3 M1 from a single third instar larva. Statistical significance was determined using ANOVA. Data are shown as the mean \pm SEM. ${ }^{*} p<0.05,{ }^{* *} p<0.01,{ }^{* * *} p<0.001$. 
of Gbb signaling leads to decreased synapse number for both MNIs and MN4Ib motoneurons, there was no correlation between NMJ area of Ib and Is in Gbb mutants (Pearson $R=0.13, n=10$, $p=0.73)$. These data are consistent with $\mathrm{Ib}$ and Is motoneurons forming synapses on the muscle without obvious competitive or cooperative interactions that shape their connectivity during development.

As described above, $\sim 30 \%$ of larval M1 muscles lack Is innervation. This natural variation provided an opportunity to examine whether growth of the coinnervating MN1-Ib motoneuron was altered at mature third instar NMJs when MNIs innervation was absent. Lack of Is innervation did not result in any change in M1 muscle surface area (coinnervation: 46,661 $\pm 1457 \mu \mathrm{m}^{2}$, $n=16$; Ib only: $48,206 \pm 1170 \mu \mathrm{m}^{2}, n=8$; $p=0.66$, ANOVA). Although the total $\mathrm{AZ}$ number was reduced at M1 in the absence of Is innervation (Ib only: $285.8 \pm 29.7$ AZs, $n=10$; coinnervation: $389.7 \pm 17.3 \mathrm{AZs}, n=13 ; F_{(5,60)}=$ $62.05, p=0.0068$; Fig. $6 A$ ), the AZ number contributed solely by the MN1-Ib input was not significantly altered whether Is was absent $(285.8 \pm$ 29.7 Ib AZs, $n=10)$ or present (329.1 $\pm 15.8 \mathrm{Ib}$ AZs, $n=13 ; F_{(5,60)}=62.05$; $p=0.66$; Fig. $6 A$ ). Likewise, no change in synaptic bouton number in MN1-Ib motoneurons was observed whether MNIs was absent $(18.0 \pm 4.3 \mathrm{Ib}$ boutons, $n=8)$ or present $(19.1 \pm 4.4 \mathrm{Ib}$ boutons, $n=16 ; F_{(5,66)}=60.73, p=0.99$; Fig. $6 B$ ). In addition, the NMJ area of MN4-Ib terminals in $G b b$ mutants was not altered if MNIs was present (52.8 \pm $\left.8.4 \mu^{2}, \quad n=10\right)$ or absent (57.4 \pm $\left.7.0 \mu \mathrm{m}^{2}, n=16 ; p=0.68\right)$. We conclude that synaptic growth of $\mathrm{Ib}$ motoneurons is not altered when Is innervation is lacking.

\section{Ablation of MNIs triggers increased evoked release from the remaining MN1-Ib input}

Although no structural compensation was observed in MN1-Ib when MNIs was absent, functional changes in neurotransmitter release could occur in the absence of increased release sites. In addition, a mismatch in neuronal activity between inputs during development could result in unique forms of plasticity compared with when a motoneuron was missing. To generate an input imbalance, MN1-Ib or MNIs GAL4 drivers were used to express several well characterized UAS transgenes that alter neuronal activity (Simpson, 2009; Venken et al., 2011; Yoshihara and Ito, 2012; Pauls et al., 2015). To decrease neurotransmitter release and synaptic output, a transgene encoding tetanus toxin light chain (UASTeTXLC) was expressed to cleave the v-SNARE n-Synaptobrevin and to eliminate evoked synaptic transmission (Sweeney et al.,
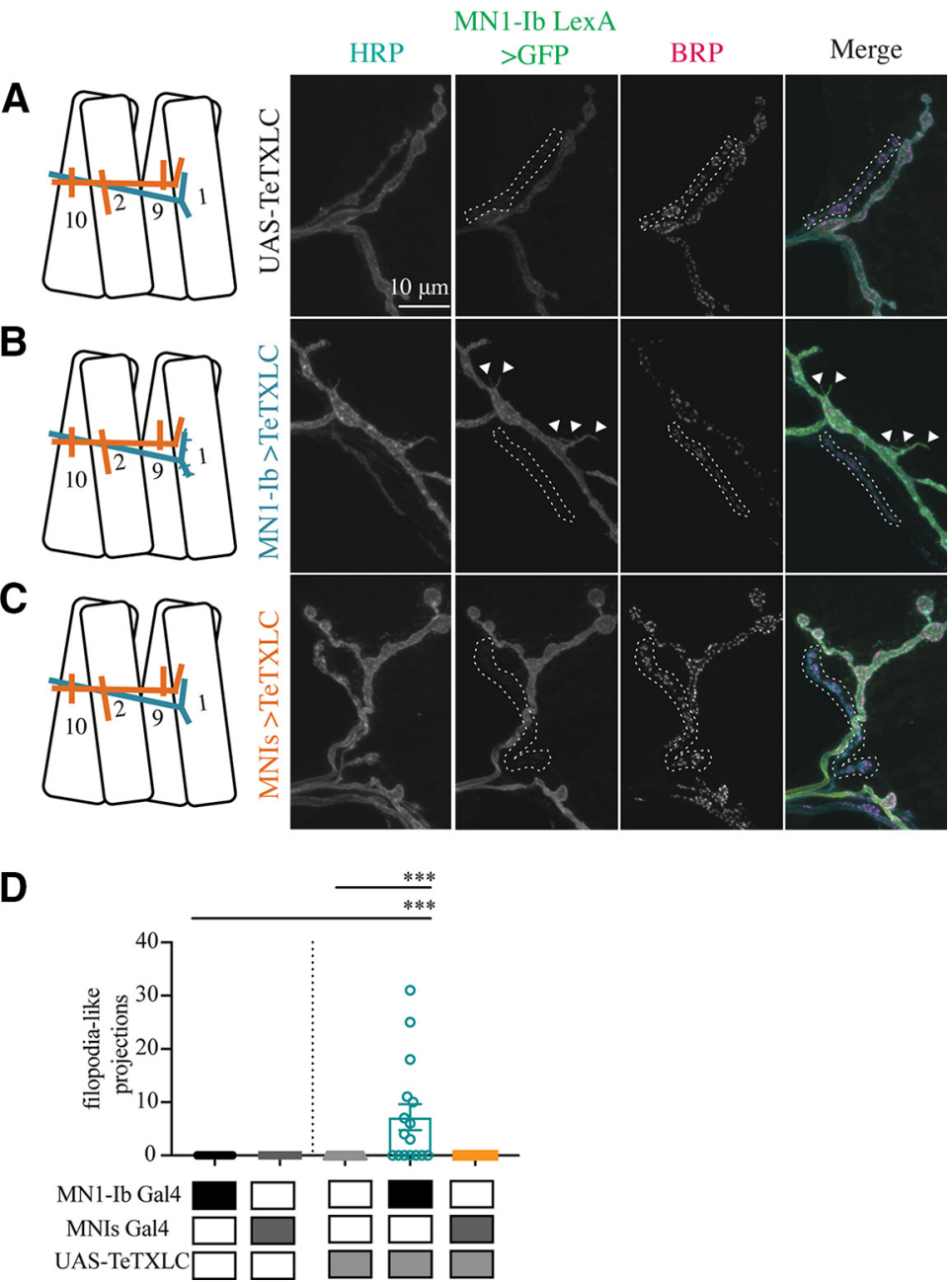

Figure 10. Morphologic consequences of silencing of MN1-Ib or MNIs. A-C, Representative confocal images of third instar larval M1 NMJs at segment A3 following immunolabeling with anti-HRP, anti-GFP, and anti-BRP in the following genotypes: UAS-TeTXLC control (A); MN1-Ib GAL4>UAS-TeTXLC (B); and MNIs GAL4>UAS-TeTXLC (C). MN1-Ib LexA>LexAop2-CD8-GFP

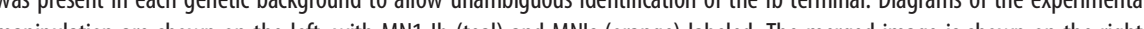
The white dashed line highlights the MNls terminal in the final three panels for each manipulation. Arrowheads in $\boldsymbol{B}$ depict GFP-positive filopodial-like projections from MN1-lb following tetanus toxin expression. Scale bars: all panels, $10 \mu \mathrm{m}$. $\boldsymbol{D}$, Quantification of filopodial-like projections in controls and following UAS-TeTXLC expression with MN1-lb or MNIs GAL4. Each data point represents quantification from segment A3 M1 from a single third instar larvae. Statistical significance was determined using ANOVA. Data are shown as mean \pm SEM. ${ }^{* *} p<0.001$.

1995). A transgene encoding a bacterial voltage-gated $\mathrm{Na}^{+}$channel (UAS-NaChBac) that enhances depolarization was used to constitutively increase neuronal excitability (Nitabach et al., 2006). To compare the effects of reduced or enhanced activity with the complete absence of each input, Reaper expression (UAS-RPR) was used to ablate MN1-Ib or MNIs. None of the manipulations altered M1 muscle surface area $\left(F_{(10,175)}=2.129, p=0.66, n=6-\right.$ 29 /genotype), indicating that muscle growth is not affected by ablation or activity changes in MN1-Ib or MNIs motoneurons (see Fig. 8A). For all experimental manipulations, the Ib motoneuron was labeled with MN1-Ib LexA, and LexAop-GFP in the background for unambiguous identification of the two inputs following immunostaining with the pan-neuronal marker anti-HRP (Ib and Is) and anti-GFP (Ib only). Synaptic development (see Figs. 8-10, 12-13), synaptic function (see Fig. 11A) and muscle contraction force (see Fig. $11 B-D$ ) were analyzed in controls and compared with $\mathrm{Ib}$ and Is motoneurons expressing the transgenes with their respective GAL4 driver. 

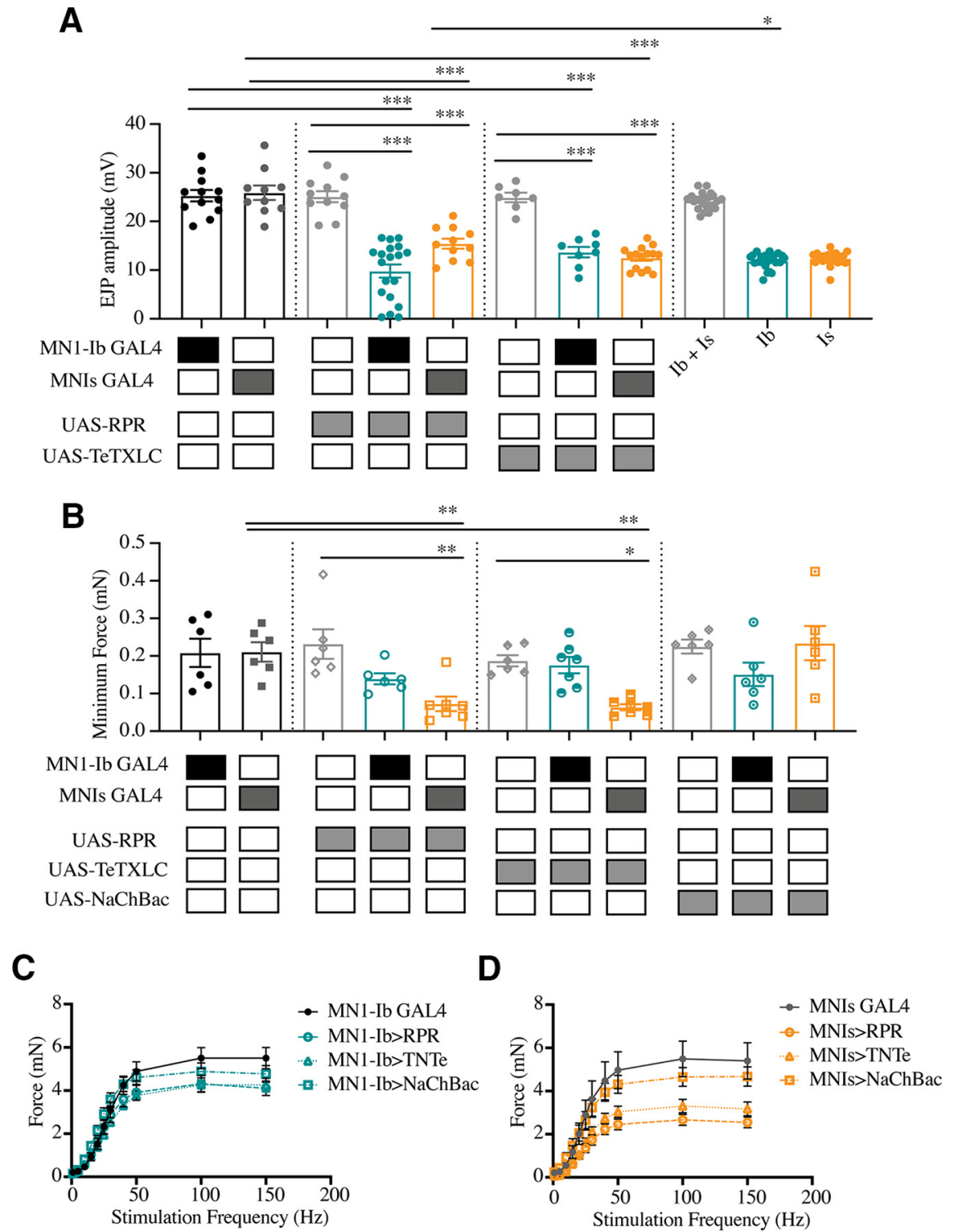

Figure 11. Electrophysiological measurements and contraction force analysis following manipulations of MN1-lb or MNIs. A, EJP amplitudes recorded from third instar larval M1 muscles in segment $A 3$ of the indicated genotypes. Each data point is the average of at least 20 EPs recorded from each larva. Shaded boxes under each bar indicate the genotypes for each group, with control GAL4 driver lines alone (MN1-Ib, MNIs), control UAS transgenes alone (UAS-RPR, UAS-TeTXLC), and experimental crosses of MN1-Ib GAL4 (teal) or MNIs GAL4 (orange) to each transgene. The final three columns on the right show results from dual intracellular recordings in controls using the minimal stimulation protocol where either MN1-lb or MNIs motoneurons were active $(\mathrm{Ib}+\mathrm{Is})$, or MN1-Ib (Ib) or MNIs (IS) were independently isolated. $\boldsymbol{B}$, Minimum contraction force in third instar larvae of the indicated genotypes. Six replicate contractions per genotype were generated per recording. Shaded boxes under each bar indicate the genotypes for each group, with control GAL4 driver lines alone (MN1-lb, MNIs), control UAS transgenes alone (UAS-RPR, UAS-TeTXLC, UAS-NaChBac), and experimental crosses of MN1-lb GAL4 (teal) or MNIs GAL4 (orange) to each transgene. C, Force-frequency curves for MN1-lb GAL4 controls and the indicated experimental genotypes. Data points represent six replicate contractions elicited at each frequency from six to seven third instar larvae. D, Force-frequency curves for MN1-Is GAL4 controls and the indicated experimental genotypes. Data points represent six replicate contractions elicited at each frequency from six to seven third instar larvae. Statistical significance was determined using ANOVA. Data are shown as the mean \pm SEM. ${ }^{*} p<0.05,{ }^{* *} p<0.01,{ }^{* * *} p<0.001$.

We first examined whether the ablation of each motoneuron subclass altered the synaptic structure of the remaining input. The expression of UAS-RPR with either MN1-Ib GAL4 or MNIs GAL4 resulted in the elimination of the respective motoneuron compared with controls (Fig. $7 A-E$ ). Membrane fragments immunopositive for GFP from the MN1-Ib LexA, LexAop-GFP labeling were often observed near M1 in MN1-Ib GAL4 $>$ UAS-RPR larvae (Fig. 7D), suggesting that cell death and membrane fragmentation occurred after the initial stages of axonal pathfinding. Genetic ablation of the Is motoneuron in MNIs GAL4>UASRPR larvae did not result in a change in MN1-Ib AZ $\left(F_{(10,183)}=52.42, p=0.999\right.$; see Fig. $9 A)$ or bouton number $\left(F_{(10,185)}=\right.$ 29.99, $p=0.999$; see Fig. $9 B)$, similar to conditions where MNIs naturally failed to innervate $\mathrm{M} 1$ in control larvae.

To determine whether MN1-Ib altered its functional properties without changes in the number of release sites, electrophysiology was performed at third instar M1 muscles to measure Ib-evoked release when Is innervation was present in controls versus when Is was ablated with UASRPR. Although no structural changes were identified at MN1-Ib NMJs, a functional change in the output of the motoneuron was observed. The evoked EJP response triggered by MN1-Ib activation was increased $24 \%$ at M1 muscles when MNIs input was ablated $(15.4 \pm 1.0 \mathrm{mV}, n=11)$ versus when MNIs was present (11.8 \pm $1.6 \mathrm{mV}, n=22 ; F_{(10,58)}=5.30, p=0.05$; see Fig. 11A). This compensation did not result in complete recovery of the evoked output observed when both inputs were present (control Is-GAL4: $25.9 \pm 1.5 \mathrm{mV}$, $n=10$ ). In addition, contractile force was still decreased following MNIs ablation, as previously noted (Fig. $4 E-G$; also see Fig. $11 B, D)$. These data suggest that the muscle is capable of detecting loss of the Is input and increasing synaptic output from the remaining $\mathrm{Ib}$ motoneuron, resulting in a partial rescue of muscle excitability.

To examine whether Is motoneurons displayed similar functional compensation, genetic ablation of the Ib motoneuron was performed using MN1-Ib GAL4; UASRPR. As previously described, M1 occasionally lacked MNIs input because of natural variation in controls. As such, MN1-Ib ablation resulted in M1 having no synaptic innervation $(42 \%)$ or only MNIs innervation (58\%). Larvae with Is innervation at M1 following ablation of the Ib motoneuron displayed a dramatic decrease in the number of total AZs (control: 332.1 \pm 13.5 AZs, $n=25$; Ib > RPR: $67.7 \pm 8.4$ AZs, $n=11 ; F_{(10,183)}=35.17, p<0.0001$; Fig. $8 B$ ) and synaptic boutons (control: $28.8 \pm 1.4$, $n=25 ; \quad \mathrm{Ib}>\mathrm{RPR}: 11.0 \pm 2.1, \quad n=11$; $F_{(10,183)}=19.47, p<0.0001$; Fig. $\left.8 C\right)$, given the larger number of synapses normally contributed by the MN1-Ib input. However, the loss of MN1-Ib did not trigger changes in the number of Is AZs (Fig. 9C) or Is synaptic boutons (Fig. 9D) compared with Is innervation when MN1-Ib was present. In contrast to the functional increase in evoked release in tonic Ib neurons following ablation of Is, the loss of MN1-Ib did not trigger a compensatory increase in evoked output from the remaining MNIs motoneuron (see Fig. 11A). We conclude that compensatory structural or functional changes do not occur in the 
phasic Is input following loss of the tonic Ib motoneuron at M1. In contrast, loss of Is results in functional changes in the coinnervating Ib input that partially compensates for the reduced evoked response.

\section{Imbalances in MN1-Ib or MNIs neuronal activity reveal structural plasticity of tonic Ib inputs}

To examine the consequences of activity perturbations between Ib and Is motoneurons at M1 NMJs during development, manipulations were performed to increase or decrease synaptic output of one of the two neurons. Expression of UAS-TeTXLC in either the Ib or Is input blocked evoked synaptic transmission from the affected motoneuron, reducing EJP amplitude recorded physiologically to the level observed when only the Ib or Is motoneuron were recruited during minimal stimulation (see Fig. 11A). Silencing the Is motoneuron in MNIs GAL4; UASTeTXLC larvae resulted in structural changes at the NMJ (Fig. 10A,C), with an increase in the total number of $\mathrm{AZs}$ (control: $396.3 \pm 14.6$ AZs, $n=15$; MNIs $>$ TeTXLC: $\quad 465.0 \pm 14.7 \quad$ AZs, $n=18$; $F_{(10,183)}=35.17, p=0.0187$; Fig. $\left.8 B\right)$ and synaptic boutons (control: 41.2 \pm 2.7 , $n=15 ; \quad$ Is $>$ TeTXLC: $50.9 \pm 2.7, n=18$; $F_{(10,183)}=19.47, p=0.028$; Fig. $\left.8 C\right)$. This enhanced synaptic growth was due to increases occurring in the coinnervating MN1-Ib input (Fig. 9A,B), with no changes observed in the affected MNIs (Fig. 9C,D). In particular, MN1-Ib displayed a large increase in $\mathrm{AZ}$ number when Is was silenced compared with when Is was present or ablated (Is present: $314 \pm 12.9, n=15$; Is ablated: $326.6 \pm 14.4$, $n=14$; Is silenced: $402.7 \pm 13.0, n=18$; $\left.F_{(10,183)}=35.17, p=0.0187\right)$. Although the number of release sites increased in MN1Ib following the silencing of MNIs (MNIs>TeTXLC), electrophysiology (Fig. 11A) and contraction (Fig. $11 B, D$ ) assays indicated that these structural changes were insufficient to induce increased excitability or contractility of the muscle. We conclude that the complete absence of the Is input leads to a functional increase in release from the coinnervating tonic Ib input without a change in the number of release sites. In contrast, when Is is present but functionally silent, the Ib input displays a distinct response with a structural change that includes more release sites, but the overall functional output of the motoneuron remains unaltered.

We next examined the consequences of silencing MN1-Ib activity with TeTXLC (Fig. 10A,B). Similar to when MN1-Ib was ablated, the coinnervating MNIs did not display structural (Fig. 9C,D) or functional (Fig. $11 A-C$ ) compensation, indicating the Is motoneuron is less capable of compensatory synaptic plasticity when the coinnervating Ib motoneuron is ablated or silenced. In contrast to the lack of change in the Is input, silencing MN1-Ib ( $\mathrm{Ib}>$ TeTXLC) triggered several structural changes to its own morphology. First, a striking reduction in $\mathrm{AZ}$ number was found, with a $30 \%$ decrease in release sites in MN1-Ib motoneurons lacking evoked transmission (UASTeTXLC: $333.6 \pm 21.2$ AZs, $n=14$; MN1-Ib GAL4: $281.6 \pm$ 11.9 AZs, $n=25$; MN1-Ib>TeTXLC: $215.3 \pm 13.6$ AZs, $n=15$; $F_{(10,183)}=52.42, p=0.001 ;$ Fig. $\left.9 A\right)$. Second, there was a change in the anatomy of the MN1-Ib axon at the NMJ, with the appearance of synaptic filopodial-like protrusions (Fig. 10A-C). This phenotype was never observed in controls (average protrusions per MN1-Ib NMJ: UAS-TeTXLC: 0, $n=14$; MN1-Ib GAL4: 0, $n=25$; MN1-Ib>TeTXLC: $7.2 \pm$ 2.4, $n=16 ; F_{(4,83)}=9.921, p=0.0001$; Fig. 10D). Similar filopodiallike protrusions were described previously at early first instar NMJs during the initial stages of synapse formation in wild-type animals, but never at mature third instar NMJs (Akbergenova et al., 2018). Such protrusions were not observed in silenced Is motoneurons or in $\mathrm{Ib}$ motoneurons following Is silencing (Fig. $10 A-D$ ), indicating that $\mathrm{MN1}-\mathrm{Ib}$ and MNIs react differently to changes in their own activity. 


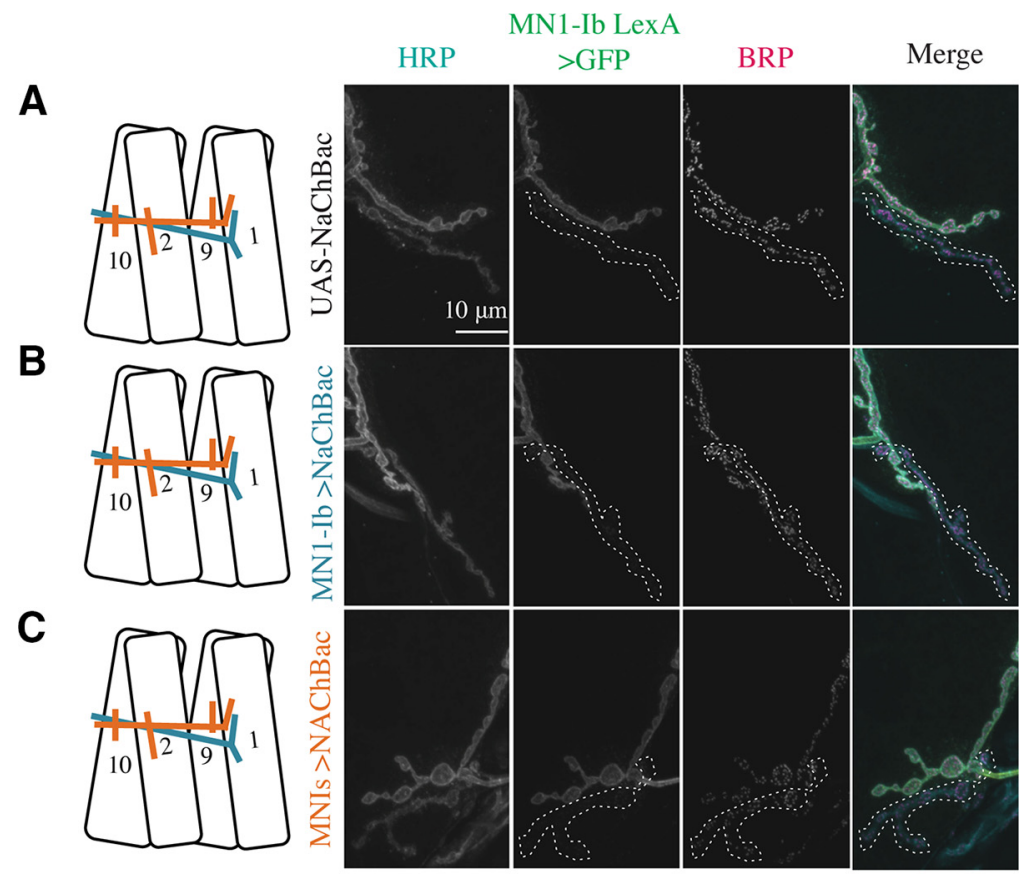

D

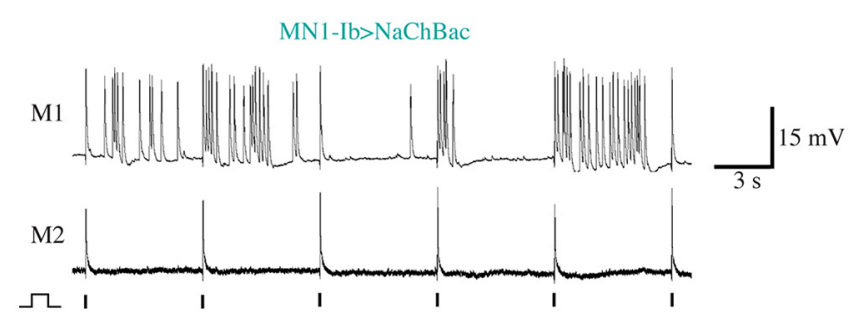

$E$

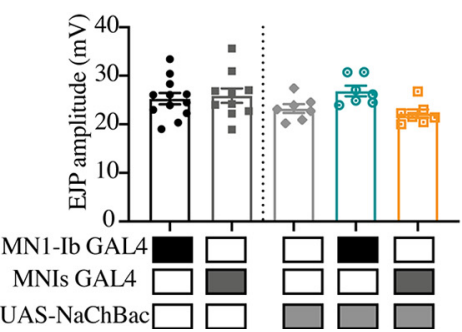

Figure 13. Chronic increases in MN1-lb or MNIs activity do not impact NMJ morphology or synaptic release. $\boldsymbol{A}-\boldsymbol{C}$, Representative confocal images of third instar larval M1 NMJs at segment A3 following immunolabeling with antiHRP, anti-GFP, and anti-BRP in the following genotypes: UAS-NaChBac control (A); MN1-lb GAL4>UAS-NaChBac (B); and MNIs GAL4> UAS-NaChBac (C). MN1-Ib LexA>LexAop2-CD8-GFP was present in each genetic background to allow unambiguous identification of the $\mathrm{lb}$ terminal. Diagrams of the experimental manipulation are shown on the left, with MN1-lb (teal) and MNIs (orange) labeled. The merged image is shown on the right. The white dashed line highlights the MNls terminal in the final three panels for each manipulation. Scale bars: all panels, $10 \mu \mathrm{m}$. $\boldsymbol{D}$, Representative dual intracellular recordings from $\mathrm{M} 1$ and $\mathrm{M} 2$ in $\mathrm{MN1}-\mathrm{Ib}>\mathrm{NaChBac}$ third instar larvae during $0.2 \mathrm{~Hz}$ stimulation. Note the train of EPs following a single stimulus at M1 compared with M2. Vertical lines below the M2 recordings indicate the timing of nerve stimulation. $\boldsymbol{E}$, EJP amplitudes recorded from third instar larval M1 muscles in segment A3 of the indicated genotypes. Each data point is the average of at least 20 EJPs recorded from each larva. Statistical significance was determined using ANOVA. No statistical difference was found across genotypes. Data are shown as the mean \pm SEM.

A final morphologic change at silenced MN1-Ib NMJs was a decrease in postsynaptic SSR membrane revealed by anti-DLG staining (Fig. 12A-D). SSR volume compared with presynaptic $\mathrm{NMJ}$ volume (anti-HRP staining) was reduced by $50 \%$ on average at MN1-Ib $>$ TeTXLC NMJs compared with controls $\left(F_{(2,54)}=\right.$ 11.70, $p=0.0079$; Fig. 12D). Together, the reduced AZ number, decreased muscle SSR volume, and increased synaptic filopodial- like protrusions suggest that silenced $\mathrm{MN1}$-Ib motoneurons maintain an immature state with reduced $\mathrm{AZ}$ formation and a failure to properly induce normal postsynaptic specializations. These defects are not observed at silenced Is phasic synapses, indicating that the Is motoneuron class is less sensitive to activity changes and any potential compensatory responses triggered from the muscle.

To determine whether enhanced activity of either of the two motoneuron subclasses would induce structural or functional changes, the $\mathrm{NaChBac}$ depolarizing $\mathrm{Na}^{+}$channel was expressed in either MN1-Ib or MNIs (Fig. $13 A-C)$. NaChBaC expression has been previously demonstrated to enhance membrane depolarization by increasing $\mathrm{Na}^{+}$ conductance (Pauls et al., 2015). Consistent with enhanced excitability and increased burst spiking in affected motoneurons, trains of EJPs in response to a single stimulus were often recorded from M1 in larvae expressing $\mathrm{NaChBac}$ ( $50 \%$ of MN1-Ib motoneurons; 38\% of MN1s motoneurons; Fig. 13D). Although expression of the channel-enhanced excitability, it did not result in structural (Figs. 8,9 ) or functional (Figs. 11, 13E) changes in synaptic properties of $\mathrm{MN1}-\mathrm{Ib}>\mathrm{NaChBac}$ or MNIs $>\mathrm{NaChBac}$ larvae. No alterations of the $\mathrm{Ib}$ or Is input were observed in either condition. Similarly, increased activity in either motoneuron class did not trigger any obvious structural competition between the inputs. We conclude that NMJ plasticity is more sensitive to manipulations that reduce presynaptic release versus those that enhance membrane excitability, and that these changes preferentially manifest within the tonic Ib subclass of motoneurons.

\section{Discussion}

To characterize how changes in the presence or activity of tonic Ib versus phasic Is motoneurons alter NMJ development and function in Drosophila, we identified GAL4 drivers specific for each class that innervate M1 and used them to alter the balance of input to the muscle. Our data indicate that $\mathrm{Ib}$ and Is motoneurons largely form independent inputs that make similar contributions to muscle excitability and contractile force. The Ib subclass was capable of structural and functional changes following manipulations that altered their output or that of the coinnervating Is motoneuron (Fig. 14A-D). These changes were observed only during conditions when neuronal activity was decreased or when the Is input was ablated. Functional increases in evoked release without enhanced synapse number were observed in Ib motoneurons following ablation of Is (Fig. 14B). In contrast, morphologic changes that increased $\mathrm{AZ}$ number without enhancing evoked release 
occurred when Is synaptic output was blocked with tetanus toxin (Fig. 14C). While $\mathrm{Ib}$ motoneurons were capable of several forms of plastic change following reduced input to the muscle, Is motoneurons were insensitive to manipulations of their own activity or that of the Ib input. Unlike the plasticity observed in Ib neurons following reduction in synaptic drive to the muscle, enhancing excitability of either the Ib or Is input was ineffective at triggering changes in either motoneuron class. These data indicate that reductions in activity from either input trigger a structural or functional change primarily from the tonic Ib motoneuron subclass.

The stereotypical connectivity found in the abdominal musculature of Drosophila larvae suggest that individual muscles normally allow synaptic innervation from only a single motoneuron of each subclass (Hoang and Chiba, 2001). However, expanded postsynaptic target choice has been observed following muscle loss induced by laser ablation or genetic mutation, with the affected Ib motoneuron targeting inappropriate nearby muscles without altering the innervation pattern of the correctly targeted Ib neuron (Sink and Whitington, 1991; Keshishian et al., 1994; Chang and Keshishian, 1996). Similarly, ablation of some motoneurons can result in axonal spouting from neighboring connections that target the deinnervated muscle (Chang and Keshishian, 1996). Misexpression of synaptic cell surface proteins can also alter target choice for some Ib and Is motoneurons (Lin and Goodman, 1994; Kose et al., 1997; Shishido et al., 1998; Ashley et al., 2019). In addition, silencing neuronal activity during development has been demonstrated to induce ectopic NMJs formed primarily by type II neuromodulatory neurons (Keshishian et al., 1994; Jarecki and Keshishian, 1995; White et al., 2001; Lnenicka et al., 2003; Mosca et al., 2005; Carrillo et al., 2010; Vonhoff and Keshishian, 2017). We did not observe any axonal sprouting onto M1 from other motoneurons that resulted in altered target choice when MN1-Ib or MNIs was ablated or silenced in our experiments. Given that M1 is the most dorsal muscle of the abdominal musculature, axons from other motoneurons are not present in the direct vicinity, so any signals released from M1 might be insufficient to attract additional innervation. We did find evidence that M1 may attempt to promote synaptic innervation when MN1-Ib was silenced with tetanus toxin. Under these conditions, the MN1-Ib terminal maintained an immature-like state with the presence of filopodial-like extensions (Fig. 14D). This effect was observed only in Ib motoneurons, highlighting differences in how Is terminals interact with or respond to signals from the muscle. We and others have observed similar filopodial-like extensions at newly forming NMJ connections in late embryos and early first instar larvae (Halpern et al., 1991; Broadie and Bate, 1993; Ritzenthaler et al., 2000; Ritzenthaler and Chiba, 2003; Kohsaka and Nose,
B Wildtype MNIs Ablation $\mathrm{MN1-Ib}$

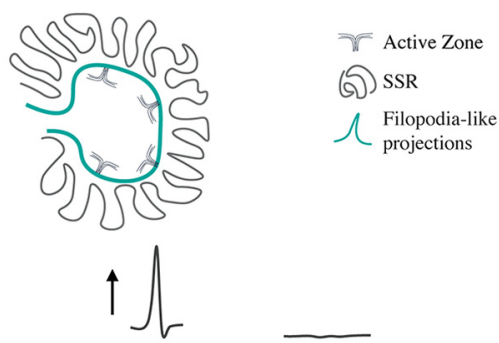

D MN1-Ib $>$ Tetanus Toxin

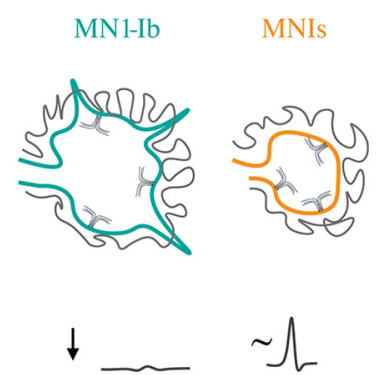

Figure 14. Summary of observed MN1-lb plasticity. $A$, In wild-type, MN1-lb and MNIs provide similar drive to muscle M1. MN1-lb forms more synaptic boutons and AZs onto M1 compared with MNIs. $\boldsymbol{B}$, Ablation of MNIs results in increased output for Pos Postsynaptic SSR development is also reduced. Presynaptic filopodia-like projections normally restricted to early first instar stage are observed at mature MN1-lb silenced terminals. No structural or functional $(\sim)$ changes occur in the coinnervating MNIs.

2009; Akbergenova et al., 2018). These presynaptic filopodial processes contain elevated levels of the Cacophony N-type $\mathrm{Ca}^{2+}$ channel and interact with GluRIIA-rich myopodia, with some progressing to form new synapses during early development (Akbergenova et al., 2018). Because of the lack of reinforcement signals caused by the absence of synaptic activity in silenced MN1-Ib motoneurons, we hypothesize that these processes fail to properly drive AZ assembly and new synapse formation. Indeed, a role for neuronal activity in regulating synaptogenic filopodial stabilization has been characterized in the Drosophila visual system (Sheng et al., 2018; Özel et al., 2019).

Many forms of plasticity, including synapse elimination at mammalian NMJs, ocular dominance plasticity, and cerebellar climbing fiber pruning, require Hebbian-like input imbalances to trigger synaptic interactions (Wiesel and Hubel, 1963, 1965; Sherman and Spear, 1982; Colman et al., 1997; Sanes and Lichtman, 1999; Walsh and Lichtman, 2003; Turney and Lichtman, 2012; Hashimoto and Kano, 2013; Tomàs et al., 2017; Wilson et al., 2019). As such, we were interested to see whether changes in the activity of $\mathrm{Ib}$ or Is motoneurons that created an imbalance between the output of the two neurons could drive unique changes compared with when one input was missing. In the case of the Ib neuron, this was indeed observed. In the absence of Is input, either because of natural variation in innervation in control animals or following ablation with UAS-RPR, there was no structural response in terms of adding additional release sites. 
However, the loss of Is triggered a functional increase in evoked release from the Ib neuron (Fig. 14B). In contrast, when an activity imbalance was created by expressing tetanus toxin in the Is neuron, Ib displayed structural plasticity that increased the number of release sites (Fig. 14C). Although the underlying molecular pathways that mediate the two distinct responses are unknown, the results suggest that the physical presence of Is likely alters the signaling systems responsible for triggering compensation in Ib motoneurons in response to reduced muscle drive.

For every manipulation we made beyond increasing excitability of the neurons, a response from the tonic Ib class was detected, while the phasic Is motoneuron displayed less plasticity. Since each muscle is innervated by a single Ib motoneuron, this plasticity may allow more robust and local regulation of muscle function. Although the Is did not show plastic change in response to manipulation of its activity or the coinnervating $\mathrm{Ib}$ in our experiments, we cannot rule out that Is neurons are capable of such plasticity but display less sensitivity to putative muscle-derived retrograde signals. Given that Is neurons innervate multiple muscles compared with Ib, it is also possible that small plastic changes occurring in Is are not synapse specific and are distributed over a larger population of AZs onto multiple muscles, resulting in little effect at any single postsynaptic target. Similar differences in homeostatic plasticity in Ib versus Is motoneurons have been described following reduced postsynaptic muscle glutamate receptor function, with the $\mathrm{Ib}$ motoneuron showing a more robust upregulation of presynaptic release compared with Is (Newman et al., 2017; Li et al., 2018; Cunningham and Littleton, 2019a,b). Although homeostatic plasticity has been observed in Is motoneurons in low extracellular $\mathrm{Ca}^{2+}$ (Genç and Davis, 2019), the elevated $P_{r}$ of Is synapses may occlude further functional increases in release output in higher $\left[\mathrm{Ca}^{2+}\right]$ normally found in larval hemolymph. Together, these results indicate that tonic Ib motoneurons express distinct plasticity mechanisms that can be triggered by reduced muscle function that are less robust or lacking in the phasic Is subclass. Whether the differential plasticity found in our study is linked directly to the tonic or phasic properties of Ib and Is motoneurons, or is under separate regulatory control, will require further investigation.

An important question moving forward is to identify mechanisms that control structural and functional plasticity in Ib motoneurons. Similarly, defining why the Is fails to respond to many of the same manipulations is poorly understood. Whether homeostatic plasticity mechanisms triggered in response to acute or chronic reduction in glutamate receptor function are also activated following the absence or functional silencing of presynaptic inputs as described here is unknown. Several molecular pathways contributing to homeostatic plasticity have been described at the NMJ (Davis, 2006, 2013; Bergquist et al., 2010; Müller et al., 2011, 2012; Müller and Davis, 2012; Younger et al., 2013; Frank, 2014; Wang et al., 2014, 2016; Davis and Müller, 2015; Gaviño et al., 2015; Kiragasi et al., 2017; Li et al., 2018; Ortega et al., 2018; Böhme et al., 2019; Goel et al., 2019; Gratz et al., 2019; Frank et al., 2020). Beyond Drosophila, studies in crustacean motor systems have shown that long-term alterations in activity can induce cell type-specific changes in tonic or phasic motoneuron structure or release properties (Atwood and Wojtowicz, 1986; Lnenicka et al., 1986, 1991; Govind and Walrond, 1989; Lnenicka and Atwood, 1989; Hong and Lnenicka, 1993; Wojtowicz et al., 1994). Given that tonic and phasic neurons are abundant in the nervous systems of both invertebrates and vertebrates (Schultz, 2001; Atwood and Karunanithi, 2002; Zucker and Regehr, 2002; Millar and Atwood, 2004; Ventimiglia and Bargmann, 2017), it will be interesting to determine whether such properties play a key role in defining their capacity for plastic change. The tools described here provide an opportunity to identify the distinct transcriptional profiles of each neuronal subclass in Drosophila to identify candidate mechanisms that mediate the differential plasticity responses of tonic Ib and phasic Is motoneurons.

\section{References}

Aberle H, Haghighi AP, Fetter RD, McCabe BD, Magalhães TR, Goodman CS (2002) wishful thinking encodes a BMP type II receptor that regulates synaptic growth in Drosophila. Neuron 33:545-558.

Akbergenova Y, Cunningham KL, Zhang YV, Weiss S, Littleton JT (2018) Characterization of developmental and molecular factors underlying release heterogeneity at Drosophila synapses. Elife 7:e38268.

Alyagor I, Berkun V, Keren-Shaul H, Marmor-Kollet N, David E, Mayseless O, Issman-Zecharya N, Amit I, Schuldiner O (2018) Combining developmental and perturbation-Seq uncovers transcriptional modules orchestrating neuronal remodeling. Dev Cell 47:38-52.e6.

Ashley J, Sorrentino V, Lobb-Rabe M, Nagarkar-Jaiswal S, Tan L, Xu S, Xiao Q, Zinn K, Carrillo RA (2019) Transsynaptic interactions between IgSF proteins DIP- $\alpha$ and Dpr10 are required for motor neuron targeting specificity. Elife 8:e42690.

Atwood HL, Karunanithi S (2002) Diversification of synaptic strength: presynaptic elements. Nat Rev Neurosci 3:497-516.

Atwood HL, Wojtowicz JM (1986) Short-term and long-term plasticity and physiological differentiation of crustacean motor synapses. Int Rev Neurobiol 28:275-362.

Atwood HL, Govind CK, Wu CF (1993) Differential ultrastructure of synaptic terminals on ventral longitudinal abdominal muscles in Drosophila larvae. J Neurobiol 24:1008-1024.

Ball RW, Warren-Paquin M, Tsurudome K, Liao EH, Elazzouzi F, Cavanagh C, An B-S, Wang T-T, White JH, Haghighi AP (2010) Retrograde BMP signaling controls synaptic growth at the NMJ by regulating trio expression in motor neurons. Neuron 66:536-549.

Berdnik D, Chihara T, Couto A, Luo L (2006) Wiring stability of the adult Drosophila olfactory circuit after lesion. J Neurosci 26:3367-3376.

Bergquist S, Dickman DK, Davis GW (2010) A hierarchy of cell intrinsic and target-derived homeostatic signaling. Neuron 66:220-234.

Berke B, Wittnam J, McNeill E, Van Vactor DL, Keshishian H (2013) Retrograde BMP signaling at the synapse: a permissive signal for synapse maturation and activity-dependent plasticity. J Neurosci 33:1793717950 .

Böhme MA, McCarthy AW, Grasskamp AT, Beuschel CB, Goel P, Jusyte M, Laber D, Huang S, Rey U, Petzoldt AG, Lehmann M, Göttfert F, Haghighi P, Hell SW, Owald D, Dickman D, Sigrist SJ, Walter AM (2019) Rapid active zone remodeling consolidates presynaptic potentiation. Nat Commun 10:1085.

Bossing T, Udolph G, Doe CQ, Technau GM (1996) The embryonic central nervous system lineages of Drosophila melanogaster. I. Neuroblast lineages derived from the ventral half of the neuroectoderm. Dev Biol 179:41-64.

Broadie KS, Bate M (1993) Development of the embryonic neuromuscular synapse of Drosophila melanogaster. J Neurosci 13:144-166.

Carrillo RA, Olsen DP, Yoon KS, Keshishian H (2010) Presynaptic activity and CaMKII modulate retrograde semaphorin signaling and synaptic refinement. Neuron 68:32-44.

Carrillo RA, Özkan E, Menon KP, Nagarkar-Jaiswal S, Lee P-T, Jeon M, Birnbaum ME, Bellen HJ, Garcia KC, Zinn K (2015) Control of synaptic connectivity by a network of drosophila IgSF cell surface proteins. Cell 163:1770-1782.

Cash S, Chiba A, Keshishian H (1992) Alternate neuromuscular target selection following the loss of single muscle fibers in Drosophila. J Neurosci 12:2051-2064

Chang TN, Keshishian H (1996) Laser ablation of Drosophila embryonic motoneurons causes ectopic innervation of target muscle fibers. J Neurosci 16:5715-5726.

Cho RW, Buhl LK, Volfson D, Tran A, Li F, Akbergenova Y, Littleton JT (2015) Phosphorylation of complexin by PKA regulates activity-dependent spontaneous neurotransmitter release and structural synaptic plasticity. Neuron 88:749-761. 
Clark MQ, Zarin AA, Carreira-Rosario A, Doe CQ (2018) Neural circuits driving larval locomotion in Drosophila. Neural Dev 13:6.

Colman H, Nabekura J, Lichtman JW (1997) Alterations in synaptic strength preceding axon withdrawal. Science 275:356-361.

Cunningham KL, Littleton JT (2019a) Neurons regulate synaptic strength through homeostatic scaling of active zones. J Cell Biol 218:1434-1435.

Cunningham KL, Littleton JT (2019b) Synaptic plasticity: close encounters of the tonic and phasic kind. Curr Biol 29:R1196-R1198.

Davis GW (2006) Homeostatic control of neural activity: from phenomenology to molecular design. Annu Rev Neurosci 29:307-323.

Davis GW (2013) Homeostatic signaling and the stabilization of neural function. Neuron 80:718-728.

Davis GW, Müller M (2015) Homeostatic control of presynaptic neurotransmitter release. Annu Rev Physiol 77:251-270.

Davis GW, DiAntonio A, Petersen SA, Goodman CS (1998) Postsynaptic PKA controls quantal size and reveals a retrograde signal that regulates presynaptic transmitter release in Drosophila. Neuron 20:305-315.

Destexhe A, Marder E (2004) Plasticity in single neuron and circuit computations. Nature 431:789-795.

Doll CA, Broadie K (2014) Impaired activity-dependent neural circuit assembly and refinement in autism spectrum disorder genetic models. Front Cell Neurosci 8:30.

Featherstone DE, Rushton E, Rohrbough J, Liebl F, Karr J, Sheng Q, Rodesch CK, Broadie K (2005) An essential Drosophila glutamate receptor subunit that functions in both central neuropil and neuromuscular junction. J Neurosci 25:3199-3208.

Foeller E, Feldman DE (2004) Synaptic basis for developmental plasticity in somatosensory cortex. Curr Opin Neurobiol 14:89-95.

Fouquet W, Owald D, Wichmann C, Mertel S, Depner H, Dyba M, Hallermann S, Kittel RJ, Eimer S, Sigrist SJ (2009) Maturation of active zone assembly by Drosophila Bruchpilot. J Cell Biol 186:129-145.

Frank CA (2014) Homeostatic plasticity at the Drosophila neuromuscular junction. Neuropharmacology 78:63-74.

Frank CA, Kennedy MJ, Goold CP, Marek KW, Davis GW (2006) Mechanisms underlying the rapid induction and sustained expression of synaptic homeostasis. Neuron 52:663-677.

Frank CA, James TD, Müller M (2020) Homeostatic control of Drosophila neuromuscular junction function. Synapse 74:e22133.

Gaviño MA, Ford KJ, Archila S, Davis GW (2015) Homeostatic synaptic depression is achieved through a regulated decrease in presynaptic calcium channel abundance. Elife 4:e05473.

Genç Ö, Davis GW (2019) Target-wide induction and synapse type-specific robustness of presynaptic homeostasis. Curr Biol 29:3863-3873.e2.

Goel P, Dufour Bergeron D, Böhme MA, Nunnelly L, Lehmann M, Buser C, Walter AM, Sigrist SJ, Dickman D (2019) Homeostatic scaling of active zone scaffolds maintains global synaptic strength. J Cell Biol 218:17061724.

Golovin RM, Vest J, Vita DJ, Broadie K (2019) Activity-dependent remodeling of Drosophila olfactory sensory neuron brain innervation during an early-life critical period. J Neurosci 39:2995-3012.

Gorczyca M, Augart C, Budnik V (1993) Insulin-like receptor and insulinlike peptide are localized at neuromuscular junctions in Drosophila. J Neurosci 13:3692-3704.

Govind CK, Walrond JP (1989) Structural plasticity at crustacean neuromuscular synapses. J Neurobiol 20:409-421.

Goyal L, McCall K, Agapite J, Hartwieg E, Steller H (2000) Induction of apoptosis by Drosophila reaper, hid and grim through inhibition of IAP function. EMBO J 19:589-597.

Gratz SJ, Goel P, Bruckner JJ, Hernandez RX, Khateeb K, Macleod GT, Dickman D, O'Connor-Giles KM (2019) Endogenous tagging reveals differential regulation of $\mathrm{Ca}^{2+}$ channels at single active zones during presynaptic homeostatic potentiation and depression. J Neurosci 39:2416-2429.

Guan Z, Saraswati S, Adolfsen B, Littleton JT (2005) Genome-wide transcriptional changes associated with enhanced activity in the Drosophila nervous system. Neuron 48:91-107.

Halpern ME, Chiba A, Johansen J, Keshishian H (1991) Growth cone behavior underlying the development of stereotypic synaptic connections in Drosophila embryos. J Neurosci 11:3227-3238.

Harris KP, Littleton JT (2015) Transmission, development, and plasticity of synapses. Genetics 201:345-375.
Harris RM, Pfeiffer BD, Rubin GM, Truman JW (2015) Neuron hemilineages provide the functional ground plan for the Drosophila ventral nervous system. Elife 4:e04493.

Hartenstein V, Campos-Ortega JA (1984) Early neurogenesis in wildtypeDrosophila melanogaster. Wilehm Roux Arch Dev Biol 193:308-325.

Hashimoto K, Kano M (2013) Synapse elimination in the developing cerebellum. Cell Mol Life Sci 70:4667-4680.

Hoang B, Chiba A (2001) Single-cell analysis of Drosophila larval neuromuscular synapses. Dev Biol 229:55-70.

Holtmaat A, Svoboda K (2009) Experience-dependent structural synaptic plasticity in the mammalian brain. Nat Rev Neurosci 10:647-658.

Hong SJ, Lnenicka GA (1993) Long-term changes in the neuromuscular synapses of a crayfish motoneuron produced by calcium influx. Brain Res 605:121-127.

Hourcade B, Muenz TS, Sandoz J-C, Rössler W, Devaud J-M (2010) Longterm memory leads to synaptic reorganization in the mushroom bodies: a memory trace in the insect brain? J Neurosci 30:6461-6465.

Jacobs JR, Goodman CS (1989) Embryonic development of axon pathways in the Drosophila CNS. II. Behavior of pioneer growth cones. J Neurosci 9:2412-2422.

Jan LY, Jan YN (1976) Properties of the larval neuromuscular junction in Drosophila melanogaster. J Physiol 262:189-214.

Jarecki J, Keshishian H (1995) Role of neural activity during synaptogenesis in Drosophila. J Neurosci 15:8177-8190.

Jenett A, Rubin GM, Ngo T-TB, Shepherd D, Murphy C, Dionne H, Pfeiffer BD, Cavallaro A, Hall D, Jeter J, Iyer N, Fetter D, Hausenfluck JH, Peng H, Trautman ET, Svirskas RR, Myers EW, Iwinski ZR, Aso Y, DePasquale GM, et al. (2012) A GAL4-driver line resource for Drosophila neurobiology. Cell Rep 2:991-1001.

Johansen J, Halpern ME, Johansen KM, Keshishian H (1989a) Stereotypic morphology of glutamatergic synapses on identified muscle cells of Drosophila larvae. J Neurosci 9:710-725.

Johansen J, Halpern ME, Keshishian H (1989b) Axonal guidance and the development of muscle fiber-specific innervation in Drosophila embryos. J Neurosci 9:4318-4332.

Karunanithi S, Cylinder D, Ertekin D, Zalucki OH, Marin L, Lavidis NA, Atwood HL, van Swinderen B (2020) Proportional downscaling of glutamatergic release sites by the general anesthetic propofol at Drosophila motor nerve terminals. eNeuro 7:ENEURO.0422-19.2020.

Katz LC, Shatz CJ (1996) Synaptic activity and the construction of cortical circuits. Science 274:1133-1138.

Keshishian H, Chang TN, Jarecki J (1994) Precision and plasticity during Drosophila neuromuscular development. FASEB J 8:731-737.

Kiragasi B, Wondolowski J, Li Y, Dickman DK (2017) A presynaptic glutamate receptor subunit confers robustness to neurotransmission and homeostatic potentiation. Cell Rep 19:2694-2706.

Kohsaka H, Nose A (2009) Target recognition at the tips of postsynaptic filopodia: accumulation and function of Capricious. Development 136:11271135.

Kose H, Rose D, Zhu X, Chiba A (1997) Homophilic synaptic target recognition mediated by immunoglobulin-like cell adhesion molecule Fasciclin III. Development 124:4143-4152.

Kurdyak P, Atwood HL, Stewart BA, Wu CF (1994) Differential physiology and morphology of motor axons to ventral longitudinal muscles in larval Drosophila. J Comp Neurol 350:463-472.

Lahey T, Gorczyca M, Jia XX, Budnik V (1994) The Drosophila tumor suppressor gene $\mathrm{dlg}$ is required for normal synaptic bouton structure. Neuron 13:823-835.

Lamprecht R, LeDoux J (2004) Structural plasticity and memory. Nat Rev Neurosci 5:45-54.

Landgraf M, Bossing T, Technau GM, Bate M (1997) The origin, location, and projections of the embryonic abdominal motorneurons of Drosophila. J Neurosci 17:9642-9655.

Lee T (2017) Wiring the Drosophila brain with individually tailored neural lineages. Curr Biol 27:R77-R82.

Lee T, Luo L (1999) Mosaic analysis with a repressible cell marker for studies of gene function in neuronal morphogenesis. Neuron 22:451-461.

Li X, Goel P, Chen C, Angajala V, Chen X, Dickman DK (2018) Synapse-specific and compartmentalized expression of presynaptic homeostatic potentiation. Elife 7:e34338.

Lin DM, Goodman CS (1994) Ectopic and increased expression of Fasciclin II alters motoneuron growth cone guidance. Neuron 13:507-523. 
Lin DM, Auld VJ, Goodman CS (1995) Targeted neuronal cell ablation in the Drosophila embryo: pathfinding by follower growth cones in the absence of pioneers. Neuron 14:707-715.

Lnenicka GA, Atwood HL (1989) Impulse activity of a crayfish motoneuron regulated its neuromuscular synaptic properties. J Neurophysiol 61:9196.

Lnenicka GA, Keshishian H (2000) Identified motor terminals in Drosophila larvae show distinct differences in morphology and physiology. J Neurobiol 43:186-197.

Lnenicka GA, Atwood HL, Marin L (1986) Morphological transformation of synaptic terminals of a phasic motoneuron by long-term tonic stimulation. J Neurosci 6:2252-2258.

Lnenicka GA, Hong SJ, Combatti M, LePage S (1991) Activity-dependent development of synaptic varicosities at crayfish motor terminals. J Neurosci 11:1040-1048.

Lnenicka GA, Spencer GM, Keshishian H (2003) Effect of reduced impulse activity on the development of identified motor terminals in Drosophila larvae. J Neurobiol 54:337-345.

Lu Z, Chouhan AK, Borycz JA, Lu Z, Rossano AJ, Brain KL, Zhou Y, Meinertzhagen IA, Macleod GT (2016) High-Probability Neurotransmitter Release Sites Represent an Energy-Efficient Design. Curr Biol 26:25622571.

Luo L, O'Leary DDM (2005) Axon retraction and degeneration in development and disease. Annu Rev Neurosci 28:127-156.

Manning L, Heckscher ES, Purice MD, Roberts J, Bennett AL, Kroll JR, Pollard JL, Strader ME, Lupton JR, Dyukareva AV, Doan PN, Bauer DM, Wilbur AN, Tanner S, Kelly JJ, Lai S-L, Tran KD, Kohwi M, Laverty TR, Pearson JC, et al. (2012) A resource for manipulating gene expression and analyzing cis-regulatory modules in the Drosophila CNS. Cell Rep 2:1002-1013.

Marder E, Calabrese RL (1996) Principles of rhythmic motor pattern generation. Physiol Rev 76:687-717.

Marder E, Rehm KJ (2005) Development of central pattern generating circuits. Curr Opin Neurobiol 15:86-93.

Marin EC, Watts RJ, Tanaka NK, Ito K, Luo L (2005) Developmentally programmed remodeling of the Drosophila olfactory circuit. Development 132:725-737.

Marqués G, Bao H, Haerry TE, Shimell MJ, Duchek P, Zhang B, O'Connor MB (2002) The Drosophila BMP type II receptor Wishful Thinking regulates neuromuscular synapse morphology and function. Neuron 33:529543.

Marrus SB, Portman SL, Allen MJ, Moffat KG, Diantonio A (2004) Differential localization of glutamate receptor subunits at the Drosophila neuromuscular junction. J Neurosci 24:1406-1415.

Matz J, Gilyan A, Kolar A, McCarvill T, Krueger SR (2010) Rapid structural alterations of the active zone lead to sustained changes in neurotransmitter release. Proc Natl Acad Sci U S A 107:8836-8841.

Mayseless O, Berns DS, Yu XM, Riemensperger T, Fiala A, Schuldiner O (2018) Developmental coordination during olfactory circuit remodeling in Drosophila. Neuron 99:1204-1215.e5.

McCabe BD, Marqués G, Haghighi AP, Fetter RD, Crotty ML, Haerry TE, Goodman CS, O'Connor MB (2003) The BMP homolog Gbb provides a retrograde signal that regulates synaptic growth at the Drosophila neuromuscular junction. Neuron 39:241-254.

Melom JE, Littleton JT (2011) Synapse development in health and disease. Curr Opin Genet Dev 21:256-261.

Melom JE, Akbergenova Y, Gavornik JP, Littleton JT (2013) Spontaneous and evoked release are independently regulated at individual active zones. J Neurosci 33:17253-17263.

Millar AG, Atwood HL (2004) Crustacean phasic and tonic motor neurons. Integr Comp Biol 44:4-13.

Mosca TJ, Carrillo RA, White BH, Keshishian H (2005) Dissection of synaptic excitability phenotypes by using a dominant-negative Shaker $\mathrm{K}+$ channel subunit. Proc Natl Acad Sci U S A 102:3477-3482.

Müller M, Davis GW (2012) Transsynaptic control of presynaptic $\mathrm{Ca}^{2+}$ influx achieves homeostatic potentiation of neurotransmitter release. Curr Biol 22:1102-1108.

Müller M, Pym ECG, Tong A, Davis GW (2011) Rab3-GAP controls the progression of synaptic homeostasis at a late stage of vesicle release. Neuron 69:749-762.
Müller M, Liu KSY, Sigrist SJ, Davis GW (2012) RIM controls homeostatic plasticity through modulation of the readily-releasable vesicle pool. J Neurosci 32:16574-16585.

Nahmani M, Turrigiano GG (2014) Adult cortical plasticity following injury: recapitulation of critical period mechanisms? Neuroscience 283:4-16.

Newman ZL, Hoagland A, Aghi K, Worden K, Levy SL, Son JH, Lee LP, Isacoff EY (2017) Input-specific plasticity and homeostasis at the Drosophila larval neuromuscular junction. Neuron 93:1388-1404.e10.

Nitabach MN, Wu Y, Sheeba V, Lemon WC, Strumbos J, Zelensky PK, White BH, Holmes TC (2006) Electrical hyperexcitation of lateral ventral pacemaker neurons desynchronizes downstream circadian oscillators in the fly circadian circuit and induces multiple behavioral periods. J Neurosci 26:479-489.

Ormerod KG, LePine OK, Bhutta MS, Jung J, Tattersall GJ, Mercier AJ (2016) Characterizing the physiological and behavioral roles of proctolin in Drosophila melanogaster. J Neurophysiol 115:568-580.

Ortega JM, Genç Ö, Davis GW (2018) Molecular mechanisms that stabilize short term synaptic plasticity during presynaptic homeostatic plasticity. Elife 7:e40385.

Özel MN, Kulkarni A, Hasan A, Brummer J, Moldenhauer M, Daumann IM, Wolfenberg H, Dercksen VJ, Kiral FR, Weiser M, Prohaska S, von Kleist M, Hiesinger PR (2019) Serial synapse formation through filopodial competition for synaptic seeding factors. Dev Cell 50:447-461.e8.

Pauls D, von Essen A, Lyutova R, van Giesen L, Rosner R, Wegener C, Sprecher SG (2015) Potency of transgenic effectors for neurogenetic manipulation in Drosophila larvae. Genetics 199:25-37.

Peled ES, Isacoff EY (2011) Optical quantal analysis of synaptic transmission in wild-type and rab3-mutant Drosophila motor axons. Nat Neurosci 14:519-526.

Pérez-Moreno JJ, O’Kane CJ (2019) GAL4 drivers specific for type Ib and type Is motor neurons in Drosophila. G3 (Bethesda) 9:453-462.

Petersen SA, Fetter RD, Noordermeer JN, Goodman CS, DiAntonio A (1997) Genetic analysis of glutamate receptors in Drosophila reveals a retrograde signal regulating presynaptic transmitter release. Neuron 19:1237-1248

Petzoldt AG, Lee Y-H, Khorramshahi O, Reynolds E, Plested AJR, Herzel H, Sigrist SJ (2014) Gating characteristics control glutamate receptor distribution and trafficking in vivo. Curr Biol 24:2059-2065.

Qin G, Schwarz T, Kittel RJ, Schmid A, Rasse TM, Kappei D, Ponimaskin E, Heckmann M, Sigrist SJ (2005) Four different subunits are essential for expressing the synaptic glutamate receptor at neuromuscular junctions of Drosophila. J Neurosci 25:3209-3218.

Rasse TM, Fouquet W, Schmid A, Kittel RJ, Mertel S, Sigrist CB, Schmidt M, Guzman A, Merino C, Qin G, Quentin C, Madeo FF, Heckmann M, Sigrist SJ (2005) Glutamate receptor dynamics organizing synapse formation in vivo. Nat Neurosci 8:898-905.

Ritzenthaler S, Chiba A (2003) Myopodia (postsynaptic filopodia) participate in synaptic target recognition. J Neurobiol 55:31-40.

Ritzenthaler S, Suzuki E, Chiba A (2000) Postsynaptic filopodia in muscle cells interact with innervating motoneuron axons. Nat Neurosci 3:10121017.

Rodal AA, Blunk AD, Akbergenova Y, Jorquera RA, Buhl LK, Littleton JT (2011) A presynaptic endosomal trafficking pathway controls synaptic growth signaling. J Cell Biol 193:201-217.

Sánchez-Soriano N, Prokop A (2005) The influence of pioneer neurons on a growing motor nerve in Drosophila requires the neural cell adhesion molecule homolog FasciclinII. J Neurosci 25:78-87.

Sanes JR, Lichtman JW (1999) Development of the vertebrate neuromuscular junction. Annu Rev Neurosci 22:389-442.

Schmid A, Chiba A, Doe CQ (1999) Clonal analysis of Drosophila embryonic neuroblasts: neural cell types, axon projections and muscle targets. Development 126:4653-4689.

Schmid A, Hallermann S, Kittel RJ, Khorramshahi O, Frölich AMJ, Quentin C, Rasse TM, Mertel S, Heckmann M, Sigrist SJ (2008) Activity-dependent site-specific changes of glutamate receptor composition in vivo. Nat Neurosci 11:659-666.

Schubiger M, Wade AA, Carney GE, Truman JW, Bender M (1998) Drosophila EcR-B ecdysone receptor isoforms are required for larval molting and for neuron remodeling during metamorphosis. Development 125:2053-2062.

Schultz W (2001) Reward signaling by dopamine neurons. Neuroscientist 7:293-302. 
Schuster CM, Ultsch A, Schloss P, Cox JA, Schmitt B, Betz H (1991) Molecular cloning of an invertebrate glutamate receptor subunit expressed in Drosophila muscle. Science 254:112-114.

Sheng C, Javed U, Gibbs M, Long C, Yin J, Qin B, Yuan Q (2018) Experience-dependent structural plasticity targets dynamic filopodia in regulating dendrite maturation and synaptogenesis. Nat Commun 9:3362.

Shepherd D, Sahota V, Court R, Williams DW, Truman JW (2019) Developmental organization of central neurons in the adult Drosophila ventral nervous system. J Comp Neurol 527:2573-2598.

Sherman SM, Spear PD (1982) Organization of visual pathways in normal and visually deprived cats. Physiol Rev 62:738-855.

Shishido E, Takeichi M, Nose A (1998) Drosophila synapse formation: regulation by transmembrane protein with Leu-rich repeats, CAPRICIOUS. Science 280:2118-2121.

Sigrist SJ, Reiff DF, Thiel PR, Steinert JR, Schuster CM (2003) Experience-dependent strengthening of Drosophila neuromuscular junctions. J Neurosci 23:6546-6556.

Simpson JH (2009) Mapping and manipulating neural circuits in the fly brain. In: Genetic dissection of neural circuits and behavior, Chap 3: Advances in Genetics, Vol 65 (Goodwin SF, ed), pp 79-143. Amsterdam: Elsevier.

Sink H, Whitington PM (1991) Early ablation of target muscles modulates the arborisation pattern of an identified embryonic Drosophila motor axon. Development 113:701-707.

Stocker B, Bochow C, Damrau C, Mathejczyk T, Wolfenberg H, Colomb J, Weber C, Ramesh N, Duch C, Biserova NM, Sigrist S, Pflüger H-J (2018) Structural and molecular properties of insect type II motor axon terminals. Front Syst Neurosci 12:5.

Sweeney ST, Broadie K, Keane J, Niemann H, O’Kane CJ (1995) Targeted expression of tetanus toxin light chain in Drosophila specifically eliminates synaptic transmission and causes behavioral defects. Neuron 14:341-351.

Tapia JC, Wylie JD, Kasthuri N, Hayworth KJ, Schalek R, Berger DR, Guatimosim C, Seung HS, Lichtman JW (2012) Pervasive synaptic branch removal in the mammalian neuromuscular system at birth. Neuron 74:816-829.

Technau G, Heisenberg M (1982) Neural reorganization during metamorphosis of the corpora pedunculata in Drosophila melanogaster. Nature 295:405-407.

Thomas JB, Bastiani MJ, Bate M, Goodman CS (1984) From grasshopper to Drosophila: a common plan for neuronal development. Nature 310:203207.

Tomàs J, Garcia N, Lanuza MA, Santafé MM, Tomàs M, Nadal L, Hurtado E, Simó A, Cilleros V (2017) Presynaptic membrane receptors modulate ACh release, axonal competition and synapse elimination during neuromuscular junction development. Front Mol Neurosci 10:132.

Truman JW (1990) Metamorphosis of the central nervous system of Drosophila. J Neurobiol 21:1072-1084.

Turney SG, Lichtman JW (2012) Reversing the outcome of synapse elimination at developing neuromuscular junctions in vivo: evidence for synaptic competition and its mechanism. PLoS Biol 10:e1001352.

Venken KJT, Simpson JH, Bellen HJ (2011) Genetic manipulation of genes and cells in the nervous system of the fruit fly. Neuron 72:202-230.

Ventimiglia D, Bargmann CI (2017) Diverse modes of synaptic signaling, regulation, and plasticity distinguish two classes of C. elegans glutamatergic neurons. Elife 6:e31234.
Vonhoff F, Keshishian H (2017) Cyclic nucleotide signaling is required during synaptic refinement at the Drosophila neuromuscular junction. Dev Neurobiol 77:39-60.

Wagh DA, Rasse TM, Asan E, Hofbauer A, Schwenkert I, Dürrbeck H, Buchner S, Dabauvalle M-C, Schmidt M, Qin G, Wichmann C, Kittel R, Sigrist SJ, Buchner E (2006) Bruchpilot, a protein with homology to ELKS/CAST, is required for structural integrity and function of synaptic active zones in Drosophila. Neuron 49:833-844.

Walsh MK, Lichtman JW (2003) In vivo time-lapse imaging of synaptic takeover associated with naturally occurring synapse elimination. Neuron 37:67-73.

Wang T, Hauswirth AG, Tong A, Dickman DK, Davis GW (2014) Endostatin is a trans-synaptic signal for homeostatic synaptic plasticity. Neuron 83:616-629.

Wang T, Jones RT, Whippen JM, Davis GW (2016) $\alpha 2 \delta-3$ is required for rapid transsynaptic homeostatic signaling. Cell Rep 16:2875-2888.

White BH, Osterwalder TP, Yoon KS, Joiner WJ, Whim MD, Kaczmarek LK, Keshishian H (2001) Targeted attenuation of electrical activity in Drosophila using a genetically modified $\mathrm{K}(+)$ channel. Neuron 31:699711.

White K, Grether ME, Abrams JM, Young L, Farrell K, Steller H (1994) Genetic control of programmed cell death in Drosophila. Science 264:677-683.

White K, Tahaoglu E, Steller H (1996) Cell killing by the Drosophila gene reaper. Science 271:805-807.

Wiesel TN, Hubel DH (1963) Effects of visual deprivation on morphology and physiology of cells in the cats lateral geniculate body. J Neurophysiol 26:978-993.

Wiesel TN, Hubel DH (1965) Comparison of the effects of unilateral and bilateral eye closure on cortical unit responses in kittens. J Neurophysiol 28:1029-1040.

Williams DW, Truman JW (2005) Remodeling dendrites during insect metamorphosis. J Neurobiol 64:24-33.

Wilson AM, Schalek R, Suissa-Peleg A, Jones TR, Knowles-Barley S, Pfister H, Lichtman JW (2019) Developmental rewiring between cerebellar climbing fibers and Purkinje cells begins with positive feedback synapse addition. Cell Rep 29:2849-2861.e6.

Wojtowicz JM, Marin L, Atwood HL (1994) Activity-induced changes in synaptic release sites at the crayfish neuromuscular junction. J Neurosci 14:3688-3703

Yoshihara M, Ito K (2012) Acute genetic manipulation of neuronal activity for the functional dissection of neural circuits-a dream come true for the pioneers of behavioral genetics. J Neurogenet 26:43-52.

Yoshihara M, Adolfsen B, Galle KT, Littleton JT (2005) Retrograde signaling by Syt 4 induces presynaptic release and synapse-specific growth. Science 310:858-863.

Younger MA, Müller M, Tong A, Pym EC, Davis GW (2013) A presynaptic ENaC channel drives homeostatic plasticity. Neuron 79:1183-1196.

Yu H-H, Kao C-F, He Y, Ding P, Kao J-C, Lee T (2010) A complete developmental sequence of a Drosophila neuronal lineage as revealed by twinspot MARCM. PLoS Biol 8:e1000461.

Zito K, Parnas D, Fetter RD, Isacoff EY, Goodman CS (1999) Watching a synapse grow: noninvasive confocal imaging of synaptic growth in Drosophila. Neuron 22:719-729.

Zucker RS, Regehr WG (2002) Short-term synaptic plasticity. Annu Rev Physiol 64:355-405. 